\title{
Botswana: Statistical Appendix
}

This Statistical Appendix for Botswana was prepared by a staff team of the International Monetary Fund as background documentation for the periodic consultation with the member country. It is based on the information available at the time it was completed on May 13, 2005. The views expressed in this document are those of the staff team and do not necessarily reflect the views of the government of Botswana or the Executive Board of the IMF.

The policy of publication of staff reports and other documents by the IMF allows for the deletion of market-sensitive information.

To assist the IMF in evaluating the publication policy, reader comments are invited and may be sent by e-mail to publicationpolicy@imf.org.

Copies of this report are available to the public from

International Monetary Fund • Publication Services $70019^{\text {th }}$ Street, N.W. • Washington, D.C. 20431

Telephone: (202) 623-7430 • Telefax: (202) 623-7201

E-mail: publications@imf.org • Internet: http://www.imf.org

Price: $\$ 15.00$ a copy

\section{International Monetary Fund Washington, D.C.}





\section{INTERNATIONAL MONETARY FUND}

\section{BOTSWANA}

\section{Statistical Appendix}

Prepared by a staff team consisting of Paul Heytens (head), Patrick Akatu, Atsushi Iimi, Jung Yeon Kim (all AFR), and Jennifer Mbabazi-Moyo (MFD).

Approved by the African Department

May 13, 2005

Contents Page

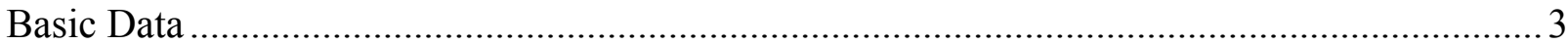

Statistical Tables

1. GDP by Type of Expenditure at Current Prices, 1998/99-2004/05 _............................ 4

2. GDP by Type of Expenditure at Constant 1993/94 Prices, 1998/99-2004/05 _................5

3. GDP by Type of Economic Activity at Current Prices, 1998/99-2004/05 .................... 6

4. GDP by Type of Economic Activity at Constant 1993/94 Prices, 1998/99-2004/05 ....... 7

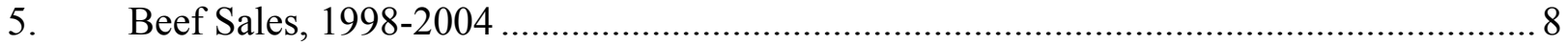

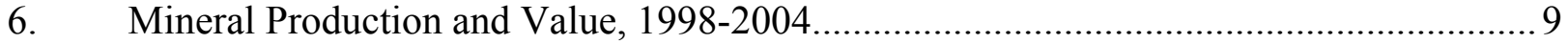

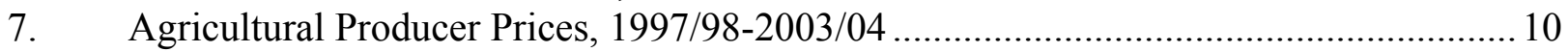

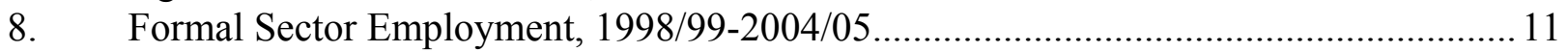

9. Statutory Minimum Hourly Wage Rates, 1998-2004 _............................................... 12

10. Average Monthly Cash Earnings by Sector, 1997-2002 ............................................ 13

11. Consumer Price Index of Tradables and Nontradables, 2000-04 ................................. 14

12. Cost of Living Index, 1991-2004 .......................................................................... 15

13. Central Government Operations, 1998/99-2005/06............................................. 16

14. Components of Central Government Revenue, 1998/99-2005/06............................ 17

15. Economic Classification of Central Government Expenditure, 1998/99-2005/06 .......... 18

16. Functional Classification of Central Government Expenditure, 1998/99-2005/06 ........ 19

17. Summary of Operations of Nonfinancial Public Enterprises, 1998-2003 ....................20

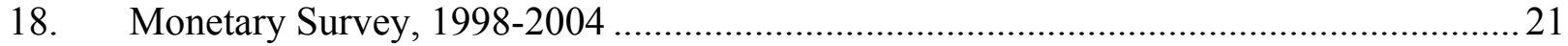

19. Summary Accounts of Bank of Botswana, 1998-2004 ............................................ 22

20. Summary Accounts of Commercial Banks, 1998-2004 ............................................. 23

21. Selected Financial Ratios and Aggregates of Commercial Banks, 1998-2004 ............. 24

22. Selected Interest Rates, 1998-2004 ................................................................ 25

23. Distribution of Commercial Bank Credit by Economic Activity, 1998-2004 ............... 26 
24. Sources of Commercial Bank Deposits, 1998-2004 .............................................. 27

25. Auctions of Bank of Botswana Certificates, 2002-04 .............................................28

26. Value of Outstanding Bank of Botswana Certificates, 2002-04 .................................29

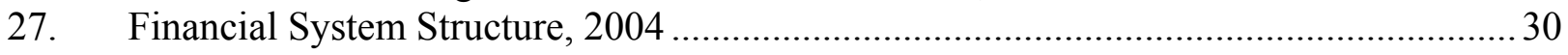

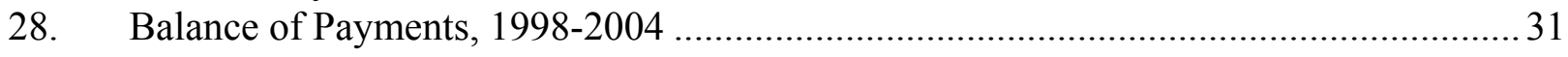

29. Value of Principal Exports and Imports, 1998-2004 ............................................. 32

30. External Trade Indices, 1998/99-2002/03 ........................................................... 33

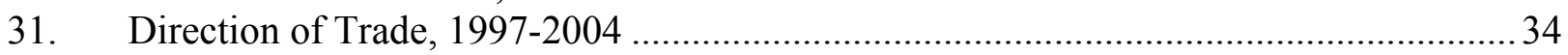

32 Public Sector External Debt, 1998-2004 …......................................................... 35

33. External Debt-Service Payments and Disbursements, 1998-2004 ............................. 36

34. Average Terms of New Public Sector External Borrowing, 1997-2004 ...................... 37

35. Developments in the Exchange Rate, 1990-2004 …............................................... 38

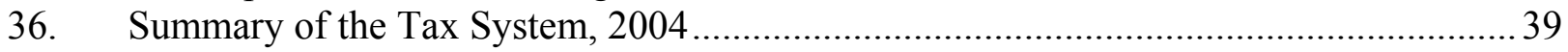


Botswana: Basic Data 1/

(In percent, unless otherwise indicated)

\begin{tabular}{lr}
\hline & \\
Land area (in square km) & 582,000 \\
Share of arable land & 0.7 \\
& \\
Population (in millions) & 1.7 \\
Share of urban population & 49.9 \\
Annual population growth & 1.0 \\
GDP per capita (US dollar) & 4,660 \\
Population below US\$ 1 a day & 23.4 \\
Gini coefficient & 0.6 \\
Unemployment rate & 23.8 \\
Life expectancy at birth (in years) & 41.4 \\
Mortality rate, infant (per 1,000 live births) & 80.0 \\
Mortality rate, under 5 (per 1,000) & 110.0 \\
Literacy rate (adult) & 78.9 \\
School enrollment, primary (male , net) & 79.2 \\
School enrollment, primary (female, net) & 82.7 \\
School enrollment, secondary (male, net) & 50.7 \\
School enrollment, secondary (female, net) & 58.5 \\
Paved roads (of total roads) & 55.0 \\
Telephone mainlines (per 1,000 people) & 87.2 \\
Water source access (urban) & 100.0 \\
Water source access (rural) & 90.0 \\
Sanitation facility access (urban) & 88.0 \\
Sanitation facility access (rural) & 43.0 \\
& \\
\hline Sources: Botswana authorities; and World Bank, World Development Indicators. \\
1/ Based on the latest available data. & \\
& \\
& \\
&
\end{tabular}


Table 1. Botswana: GDP by Type of Expenditure at Current Prices, 1998/99-2004/05 1/

\begin{tabular}{|c|c|c|c|c|c|c|c|}
\hline & 1998/99 & $1999 / 00$ & $2000 / 01$ & $2001 / 02$ & $2002 / 03$ & $2003 / 04$ & $\begin{array}{r}2004 / 05 \\
\text { Est. }\end{array}$ \\
\hline & \multicolumn{7}{|c|}{ (In millions of pula) } \\
\hline Total consumption & 13,516 & 15,366 & 17,180 & 19,860 & 22,503 & 25,167 & 27,754 \\
\hline General government & 6,579 & 7,525 & 8,742 & 10,553 & 12,167 & 13,692 & 14,949 \\
\hline Central government & 5,841 & 6,710 & 7,839 & 9,508 & 11,014 & 12,422 & 13,330 \\
\hline Local government & 738 & 815 & 903 & 1,045 & 1,154 & 1,270 & 1,619 \\
\hline Private & 6,937 & 7,841 & 8,439 & 9,308 & 10,336 & 11,475 & 12,805 \\
\hline Total investment & 7,917 & 5,084 & 5,607 & 8,351 & 10,737 & 11,967 & 11,665 \\
\hline Gross fixed capital formation & 6,263 & 6,751 & 6,898 & 7,743 & 8,736 & 9,556 & 11,253 \\
\hline Public & 3,333 & 2,935 & 3,372 & 3,824 & 4,150 & 4,274 & 4,459 \\
\hline Private & 2,930 & 3,817 & 3,526 & 3,920 & 4,586 & 5,282 & 6,793 \\
\hline Changes in stocks & 1,654 & $-1,667$ & $-1,291$ & 608 & 2,001 & 2,411 & 412 \\
\hline Net exports of goods and services & 91 & 4,896 & 6,749 & 3,826 & 3,820 & 3,007 & 4,513 \\
\hline Exports of goods and services & 10,052 & 15,318 & 17,555 & 15,564 & 16,132 & 15,864 & 16,414 \\
\hline Of which: exports of goods & 8,560 & 13,637 & 15,714 & 12,993 & 12,762 & 12,507 & 3,756 \\
\hline Imports of goods and services & $-9,961$ & $-10,422$ & $-10,806$ & $-11,738$ & $-12,313$ & $-12,857$ & $-11,901$ \\
\hline Of which: imports of goods & $-8,571$ & $-8,866$ & $-8,965$ & $-9,732$ & $-10,184$ & $-10,456$ & $-3,360$ \\
\hline Gross domestic savings 2/ & 8,008 & 9,577 & 11,456 & 12,062 & 14,212 & 14,714 & 16,574 \\
\hline Central government & 1,807 & 1,453 & 1,715 & 3,009 & 2,433 & 3,089 & 3,936 \\
\hline Other & 6,201 & 8,124 & 9,741 & 9,053 & 11,779 & 11,625 & 12,638 \\
\hline Resource gap & 91 & 4,493 & 5,849 & 3,711 & 3,475 & 2,747 & 4,909 \\
\hline \multirow[t]{2}{*}{ Total GDP } & 21,524 & 24,943 & 28,636 & 31,922 & 36,715 & 39,881 & 44,327 \\
\hline & \multicolumn{7}{|c|}{ (In percent of GDP) } \\
\hline Total consumption & 62.8 & 61.6 & 60.0 & 62.2 & 61.3 & 63.1 & 62.6 \\
\hline General government & 30.6 & 30.2 & 30.5 & 33.1 & 33.1 & 34.3 & 33.7 \\
\hline Central government & 27.1 & 26.9 & 27.4 & 29.8 & 30.0 & 31.1 & 30.1 \\
\hline Local government & 3.4 & 3.3 & 3.2 & 3.3 & 3.1 & 3.2 & 3.7 \\
\hline Private & 32.2 & 31.4 & 29.5 & 29.2 & 28.2 & 28.8 & 28.9 \\
\hline Total investment & 36.8 & 20.4 & 19.6 & 26.2 & 29.2 & 30.0 & 26.3 \\
\hline Gross fixed capital formation & 29.1 & 27.1 & 24.1 & 24.3 & 23.8 & 24.0 & 25.4 \\
\hline Public & 15.5 & 11.8 & 11.8 & 12.0 & 11.3 & 10.7 & 10.1 \\
\hline Private & 13.6 & 15.3 & 12.3 & 12.3 & 12.5 & 13.2 & 15.3 \\
\hline Changes in stocks & 7.7 & -6.7 & -4.5 & 1.9 & 5.5 & 6.0 & 0.9 \\
\hline Net exports of goods and services & 0.4 & 19.6 & 23.6 & 12.0 & 10.4 & 7.5 & 10.2 \\
\hline Exports of goods and services & 46.7 & 61.4 & 61.3 & 48.8 & 43.9 & 39.8 & 37.0 \\
\hline Of which : exports of goods & 39.8 & 54.7 & 54.9 & 40.7 & 34.8 & 31.4 & 8.5 \\
\hline Imports of goods and services & -46.3 & -41.8 & -37.7 & -36.8 & -33.5 & -32.2 & -26.8 \\
\hline Of which: imports of goods & -39.8 & -35.5 & -31.3 & -30.5 & -27.7 & -26.2 & -7.6 \\
\hline Gross domestic savings 2/ & 37.2 & 38.4 & 40.0 & 37.8 & 38.7 & 36.9 & 37.4 \\
\hline Central government & 8.4 & 5.8 & 6.0 & 9.4 & 6.6 & 7.7 & 8.9 \\
\hline Other & 28.8 & 32.6 & 34.0 & 28.4 & 32.1 & 29.1 & 28.5 \\
\hline Resource gap & 0.4 & 18.0 & 20.4 & 11.6 & 9.5 & 6.9 & 11.1 \\
\hline
\end{tabular}

Sources: Central Statistics Office; and IMF staff estimates.

1/ National accounts year beginning July 1.

2/ GDP minus consumption. 
Table 2. Botswana: GDP by Type of Expenditure at Constant 1993/94 Prices, 1998/99-2004/05 1/

\begin{tabular}{|c|c|c|c|c|c|c|c|}
\hline & 1998/99 & $1999 / 00$ & $2000 / 01$ & $2001 / 02$ & $2002 / 03$ & $2003 / 04$ & $\begin{array}{r}2004 / 05 \\
\text { Est. }\end{array}$ \\
\hline & \multicolumn{7}{|c|}{ (In millions of pula) } \\
\hline Consumption & 8,743 & 9,281 & 9,704 & 10,586 & 10,963 & 11,900 & 12,699 \\
\hline Public & 4,194 & 4,538 & 4,966 & 5,635 & 5,969 & 6,522 & 6,716 \\
\hline Private & 4,549 & 4,743 & 4,738 & 4,951 & 4,994 & 5,378 & 5,983 \\
\hline Total investment & 5,613 & 3,425 & 3,344 & 4,457 & 5,415 & 6,024 & 5,136 \\
\hline Gross fixed capital formation & 4,393 & 4,463 & 4,194 & 4,451 & 4,516 & 4,813 & 4,961 \\
\hline Of which: general government & 2,247 & 2,334 & 2,426 & 3,234 & 3,930 & $\ldots$ & $\ldots$ \\
\hline Changes in stocks & 1,220 & $-1,038$ & -850 & 7 & 900 & 1,211 & 175 \\
\hline Net exports of goods and services & -686 & 1,946 & 2,747 & 1,200 & 1,103 & 704 & 2,205 \\
\hline Exports of goods and services & 5,952 & 8,320 & 8,834 & 7,477 & 7,092 & 6,770 & 9,062 \\
\hline Imports of goods and services & $-6,638$ & $-6,374$ & $-6,087$ & $-6,278$ & $-5,989$ & $-6,065$ & $-6,857$ \\
\hline Gross domestic expenditure & 14,356 & 12,706 & 13,049 & 15,044 & 16,379 & 17,924 & 17,835 \\
\hline \multirow[t]{2}{*}{ GDP at constant prices } & 14,296 & 15,239 & 16,535 & 16,906 & 18,217 & 19,258 & 20,041 \\
\hline & \multicolumn{7}{|c|}{ (Annual percentage change) } \\
\hline Consumption & 5.9 & 6.2 & 4.6 & 9.1 & 3.6 & 8.5 & 6.7 \\
\hline Public & 5.6 & 8.2 & 9.4 & 13.5 & 5.9 & 9.3 & 3.0 \\
\hline Private & 6.1 & 4.3 & -0.1 & 4.5 & 0.9 & 7.7 & 11.2 \\
\hline Total investment & 36.1 & -39.0 & -2.4 & 33.3 & 21.5 & 11.2 & -14.7 \\
\hline Gross fixed capital formation & 18.0 & 1.6 & -6.0 & 6.1 & 1.5 & 6.6 & 3.1 \\
\hline Of which: general government & -2.3 & 3.9 & 4.0 & 33.3 & 21.5 & $\ldots$ & $\ldots$ \\
\hline Changes in stocks & 203.9 & -185.1 & -18.1 & -100.8 & $13,652.2$ & 34.6 & -85.5 \\
\hline Net exports of goods and services & -161.7 & -383.6 & 41.2 & -56.3 & -8.1 & -36.1 & 213.1 \\
\hline Exports of goods and services & -19.6 & 39.8 & 6.2 & -15.4 & -5.2 & -4.5 & 33.9 \\
\hline Imports of goods and services & 5.5 & -4.0 & -4.5 & 3.1 & -4.6 & 1.3 & 13.1 \\
\hline Gross domestic expenditure & 15.9 & -11.5 & 2.7 & 15.3 & 8.9 & 9.4 & -0.5 \\
\hline GDP at constant prices & 4.1 & 6.6 & 8.6 & 2.1 & 7.8 & 5.7 & 4.1 \\
\hline
\end{tabular}

Sources: Central Statistics Office; and IMF staff estimates.

1/ National accounts year beginning July 1 . 
Table 3. Botswana: GDP by Type of Economic Activity at Current Prices, 1998/99-2004/05 1/

\begin{tabular}{|c|c|c|c|c|c|c|c|}
\hline & 1998/99 & $1999 / 00$ & $2000 / 01$ & $2001 / 02$ & $2002 / 03$ & $2003 / 04$ & $\begin{array}{r}2004 / 05 \\
\text { Est. }\end{array}$ \\
\hline & \multicolumn{7}{|c|}{ (In millions of pula) } \\
\hline Agriculture & 654 & 665 & 755 & 792 & 871 & 931 & 994 \\
\hline Mining & 6,693 & 8,389 & 10,086 & 11,238 & 12,636 & 13,534 & 14,767 \\
\hline Manufacturing & 1,128 & 1,240 & 1,344 & 1,404 & 1,550 & 1,617 & 1,787 \\
\hline Water and electricity & 458 & 568 & 689 & 750 & 927 & 1,062 & 1,214 \\
\hline Construction & 1,360 & 1,424 & 1,563 & 1,738 & 1,976 & 2,103 & 2,356 \\
\hline Trade and hotels & 2,339 & 2,735 & 3,193 & 3,651 & 4,178 & 4,490 & 5,168 \\
\hline Transport & 814 & 935 & 1,058 & 1,151 & 1,288 & 1,404 & 1,566 \\
\hline Banking, insurance, and business services & 2,410 & 2,761 & 3,202 & 3,644 & 4,096 & 4,589 & 5,279 \\
\hline General government & 3,751 & 4,105 & 4,568 & 5,264 & 5,818 & 6,265 & 6,885 \\
\hline Social and personal services & 870 & 994 & 1,107 & 1,249 & 1,394 & 1,522 & 1,711 \\
\hline Adjustments items & 1,047 & 1,128 & 1,073 & 1,041 & 1,982 & 2,364 & 2,600 \\
\hline \multirow[t]{2}{*}{ GDP at current prices } & 21,524 & 24,943 & 28,636 & 31,922 & 36,715 & 39,881 & 44,327 \\
\hline & \multicolumn{7}{|c|}{ (In percent of total GDP) } \\
\hline Agriculture & 3.0 & 2.7 & 2.6 & 2.5 & 2.4 & 2.3 & 2.2 \\
\hline Mining & 31.1 & 33.6 & 35.2 & 35.2 & 34.4 & 33.9 & 33.3 \\
\hline Manufacturing & 5.2 & 5.0 & 4.7 & 4.4 & 4.2 & 4.1 & 4.0 \\
\hline Water and electricity & 2.1 & 2.3 & 2.4 & 2.3 & 2.5 & 2.7 & 2.7 \\
\hline Construction & 6.3 & 5.7 & 5.5 & 5.4 & 5.4 & 5.3 & 5.3 \\
\hline Trade and hotels & 10.9 & 11.0 & 11.2 & 11.4 & 11.4 & 11.3 & 11.7 \\
\hline Transport & 3.8 & 3.8 & 3.7 & 3.6 & 3.5 & 3.5 & 3.5 \\
\hline Banking, insurance, and business services & 11.2 & 11.1 & 11.2 & 11.4 & 11.2 & 11.5 & 11.9 \\
\hline General government & 17.4 & 16.5 & 16.0 & 16.5 & 15.8 & 15.7 & 15.5 \\
\hline Social and personal services & 4.0 & 4.0 & 3.9 & 3.9 & 3.8 & 3.8 & 3.9 \\
\hline Adjustments items & 4.9 & 4.5 & 3.7 & 3.3 & 5.4 & 5.9 & 5.9 \\
\hline GDP at current prices & 100.0 & 100.0 & 100.0 & 100.0 & 100.0 & 100.0 & 100.0 \\
\hline
\end{tabular}

Sources: Central Statistics Office; and IMF staff estimates.

1/ National accounts year beginning July 1. 
Table 4. Botswana: GDP by Type of Economic Activity at Constant 1993/94 Prices, 1998/99-2004/05 1/

\begin{tabular}{|c|c|c|c|c|c|c|c|}
\hline & 1998/99 & 1999/00 & $2000 / 01$ & $2001 / 02$ & $2002 / 03$ & $2003 / 04$ & $\begin{array}{r}2004 / 05 \\
\text { Est. }\end{array}$ \\
\hline & \multicolumn{7}{|c|}{ (In millions of pula) } \\
\hline Agriculture & 443 & 405 & 445 & 433 & 441 & 446 & 444 \\
\hline Mining & 4,588 & 5,142 & 6,046 & 5,865 & 6,472 & 6,918 & 7,107 \\
\hline Manufacturing & 661 & 684 & 683 & 683 & 703 & 700 & 728 \\
\hline Water and electricity & 334 & 371 & 391 & 406 & 444 & 462 & 476 \\
\hline Construction & 917 & 939 & 955 & 1,000 & 1,005 & 1,055 & 1,113 \\
\hline Trade and hotels & 1,502 & 1,596 & 1,700 & 1,840 & 1,901 & 1,957 & 2,084 \\
\hline Transport & 579 & 594 & 624 & 625 & 631 & 639 & 652 \\
\hline Banking, insurance, and business services & 1,636 & 1,707 & 1,795 & 1,922 & 1,973 & 2,071 & 2,196 \\
\hline General government & 2,333 & 2,474 & 2,641 & 2,861 & 2,965 & 3,102 & 3,258 \\
\hline Social and personal services & 618 & 645 & 663 & 705 & 724 & 769 & 808 \\
\hline Adjustment items & 684 & 681 & 614 & 567 & 957 & 1,138 & 1,177 \\
\hline GDP at constant prices & 14,296 & 15,239 & 16,555 & 16,906 & 18,217 & 19,258 & 20,041 \\
\hline \multirow[t]{2}{*}{ Of which: Non-mining private GDP } & 9,707 & 10,097 & 10,509 & 11,041 & 11,746 & 12,339 & 12,934 \\
\hline & \multicolumn{7}{|c|}{ (Annual percentage change) } \\
\hline Agriculture & -7.6 & -8.7 & 9.9 & -2.6 & 1.9 & 1.1 & -0.5 \\
\hline Mining & -2.8 & 12.1 & 17.6 & -3.0 & 10.3 & 6.9 & 2.7 \\
\hline Manufacturing & 5.7 & 3.5 & -0.3 & 0.0 & 3.1 & -0.5 & 4.0 \\
\hline Water and electricity & 12.9 & 11.3 & 5.4 & 3.7 & 9.5 & 3.9 & 3.0 \\
\hline Construction & 11.5 & 2.4 & 1.6 & 4.7 & 0.6 & 4.9 & 5.5 \\
\hline Trade and hotels & 5.6 & 6.2 & 6.5 & 8.2 & 3.4 & 2.9 & 6.5 \\
\hline Transport & 16.2 & 2.6 & 5.0 & 0.3 & 0.9 & 1.2 & 2.0 \\
\hline Banking, insurance, and business services & 9.0 & 4.3 & 5.1 & 7.1 & 2.6 & 5.0 & 6.0 \\
\hline General government & 6.3 & 6.0 & 6.7 & 8.3 & 3.7 & 4.6 & 5.0 \\
\hline Social and personal services & 7.5 & 4.4 & 2.8 & 6.2 & 2.6 & 6.2 & 5.0 \\
\hline Adjustment items & 15.5 & -0.5 & -9.9 & -7.6 & 68.7 & 19.0 & 3.4 \\
\hline GDP at constant prices & 4.1 & 6.6 & 8.6 & 2.1 & 7.8 & 5.7 & 4.1 \\
\hline Of which : Non-mining private GDP & 7.8 & 4.0 & 4.1 & 5.1 & 6.4 & 5.1 & 4.8 \\
\hline
\end{tabular}

Sources: Central Statistics Office; and IMF staff estimates.

1/ National accounts year beginning July 1. 
Table 5. Botswana: Beef Sales, 1998-20041/

\begin{tabular}{|c|c|c|c|c|c|c|c|}
\hline & 1998 & 1999 & 2000 & 2001 & 2002 & 2003 & 2004 \\
\hline \multicolumn{8}{|c|}{ Boneless beef sales by category } \\
\hline & \multicolumn{7}{|c|}{ (In thousands of tons) } \\
\hline \multirow[t]{2}{*}{ Gross sales } & 16 & 20 & 17 & 25 & 18 & 17 & 17 \\
\hline & \multicolumn{7}{|c|}{ (In millions of pula) } \\
\hline Gross sales & 213 & 254 & 253 & 386 & 260 & 246 & 275 \\
\hline \multicolumn{8}{|l|}{ Beef sales by country } \\
\hline & \multicolumn{7}{|c|}{ (In thousands of tons) } \\
\hline Total & 16 & 20 & 17 & 25 & 16 & 17 & 17 \\
\hline United Kingdom & 10 & 14 & 11 & 15 & 10 & 9 & 9 \\
\hline South Africa & 2 & 2 & 3 & 6 & 3 & 5 & 3 \\
\hline \multirow[t]{2}{*}{ Other } & 3 & 4 & 4 & 4 & 4 & 3 & 4 \\
\hline & \multicolumn{7}{|c|}{ (In millions of pula) } \\
\hline Total & 213 & 254 & 253 & 386 & 260 & 246 & 275 \\
\hline United Kingdom & 163 & 197 & 192 & 293 & 191 & 164 & 189 \\
\hline South Africa & 19 & 20 & 22 & 49 & 26 & 44 & 34 \\
\hline Other & 31 & 37 & 38 & 44 & 43 & 37 & 52 \\
\hline \multicolumn{8}{|l|}{ Memorandum item: } \\
\hline & \multicolumn{7}{|c|}{ (In thousands) } \\
\hline Total cattle processed & 140 & 123 & 185 & 120 & 144 & 135 & $\ldots$ \\
\hline
\end{tabular}

Source: Ministry of Agriculture.

1/ With effect from 2000, the financial year is aligned with the calendar year. 
Table 6. Botswana: Mineral Production and Value, 1998-2004

\begin{tabular}{|c|c|c|c|c|c|c|c|}
\hline & 1998 & 1999 & 2000 & 2001 & 2002 & 2003 & $20041 /$ \\
\hline \multicolumn{8}{|l|}{ Diamonds } \\
\hline Volume (in millions of carats) & 20.0 & 21.0 & 25.0 & 26.0 & 28.4 & 30.4 & 31.1 \\
\hline \multicolumn{8}{|l|}{ Copper-nickel matte } \\
\hline Value (in millions of pula) 2/ & 456.0 & 558.0 & 801.0 & 901.0 & 428.0 & $1,052.3$ & 952.6 \\
\hline Volume (in thousands of tons) & 37.0 & 39.0 & 46.0 & 42.0 & 45.8 & 52.0 & 33.9 \\
\hline Unit value (in pula per ton) & $12,324.3$ & $14,307.7$ & $17,413.0$ & $21,451.6$ & $9,337.0$ & $20,242.4$ & $28,118.3$ \\
\hline \multicolumn{8}{|l|}{ Coal } \\
\hline Value (in millions of pula) 2/ & 30.0 & 26.0 & 30.0 & 29.0 & 30.0 & 25.9 & 23.4 \\
\hline Volume (in thousands of tons) & 924.0 & 945.0 & 947.0 & 930.0 & 953.0 & 822.8 & 743.5 \\
\hline Unit value (in pula per ton) & 32.0 & 27.0 & 31.0 & 32.0 & 32.0 & 31.5 & 31.5 \\
\hline \multicolumn{8}{|l|}{ Soda ash } \\
\hline Value (in millions of pula) 2/ & 137.0 & 106.0 & 122.0 & 186.0 & 210.0 & 173.8 & 172.6 \\
\hline Volume (in thousands of tons) & 190.0 & 229.0 & 190.0 & 251.0 & 283.0 & 234.5 & 209.2 \\
\hline Unit value (in pula per ton) & 720.0 & 463.0 & 639.0 & 741.0 & 741.0 & 741.0 & 825.0 \\
\hline \multicolumn{8}{|l|}{ Salt } \\
\hline Value (in millions of pula) $2 /$ & 30.0 & 19.0 & 32.0 & 37.0 & 65.0 & 47.3 & 39.1 \\
\hline Volume (in thousands of tons) & 140.0 & 168.0 & 185.0 & 179.0 & 315.0 & 229.4 & 184.3 \\
\hline Unit value (in pula per ton) & 212.0 & 115.0 & 175.0 & 206.0 & 206.0 & 206.0 & 212.0 \\
\hline
\end{tabular}

Source: Central Statistics Office and Department of Mines.

1/ Data up to October, except diamonds, which cover the whole year.

2/ Estimated value of production. 
Table 7. Botswana: Agricultural Producer Prices, 1997/98-2003/04 1/ (Pula per ton)

\begin{tabular}{|c|c|c|c|c|c|c|c|}
\hline & 1997/98 & $1998 / 99$ & 1999/00 & $2000 / 01$ & $2001 / 02$ & $2002 / 03$ & $2003 / 04$ \\
\hline Sorghum & 422.0 & 486.0 & 574.3 & 548.0 & $1,200.0$ & $\ldots$ & $\ldots$ \\
\hline White maize & 437.0 & 466.0 & 643.6 & 508.0 & 933.0 & $\ldots$ & $\ldots$ \\
\hline Pulses & 797.0 & 783.0 & 875.5 & $2,832.0$ & $3,908.0$ & $\ldots$ & $\ldots$ \\
\hline Sunflower seeds & 625.0 & 625.0 & 727.5 & 712.5 & $1,531.3$ & $\ldots$ & $\ldots$ \\
\hline Shelled groundnuts & $1,356.0$ & $1,356.0$ & $1,491.4$ & $1,642.0$ & $2,365.5$ & $\ldots$ & $\ldots$ \\
\hline
\end{tabular}

Source: Botswana Agricultural Marketing Board.

1/ Crop year beginning April 1. 
Table 8. Botswana: Formal Sector Employment, 1998/99-2004/05 1/ (Number of employees, unless otherwise indicated)

\begin{tabular}{|c|c|c|c|c|c|c|c|}
\hline & $1998 / 99$ & $1999 / 00$ & $2000 / 01$ & $2001 / 02$ & $2002 / 03$ & $2003 / 04$ & $2004 / 052$ \\
\hline Private and parastatal & 168,700 & 173,900 & 180,100 & 182,193 & 169,519 & 174,483 & 178,854 \\
\hline Private & 153,200 & 160,000 & 166,600 & 155,062 & 155,936 & 160,861 & 166,759 \\
\hline Parastatal & 15,500 & 13,900 & 13,500 & 13,564 & 13,583 & 13,622 & 12,095 \\
\hline Agriculture & 5,400 & 5,800 & 6,000 & 6,206 & 5,975 & 6,264 & 5,262 \\
\hline Mining and quarrying & 8,300 & 7,900 & 8,300 & 6,962 & 7,508 & 7,968 & 9,705 \\
\hline Manufacturing & 28,000 & 29,800 & 30,900 & 30,287 & 29,789 & 30,169 & 32,621 \\
\hline Electricity and water & 2,700 & 2,900 & 2,900 & 2,752 & 3,064 & 2,825 & 2,515 \\
\hline Construction & 27,500 & 27,300 & 28,500 & 28,785 & 28,895 & 29,750 & 25,347 \\
\hline Commerce & 44,500 & 47,600 & 49,900 & 53,527 & 53,792 & 55,288 & 56,164 \\
\hline Transport and communications & 9,800 & 9,900 & 10,100 & 9,854 & 10,136 & 10,277 & 12,700 \\
\hline Finance and business services & 17,200 & 18,300 & 19,200 & 18,266 & 18,263 & 19,025 & 21,476 \\
\hline Community and personal services & 4,200 & 4,300 & 4,300 & 5,249 & 5,327 & 5,796 & 5,177 \\
\hline Education & 5,600 & 6,200 & 6,500 & 6,741 & 6,772 & 7,121 & 7,888 \\
\hline General government & 104,000 & 105,200 & 106,400 & 105,156 & 109,380 & 110,900 & 117,533 \\
\hline Central government & 84,900 & 84,500 & 85,400 & 83,077 & 86,958 & 87,700 & 94,753 \\
\hline Local government & 19,100 & 20,700 & 21,000 & 22,079 & 22,422 & 23,200 & 22,780 \\
\hline Total & 272,700 & 279,100 & 286,500 & 287,349 & 278,899 & 285,382 & 296,387 \\
\hline \multicolumn{8}{|l|}{ Memorandum item: } \\
\hline $\begin{array}{l}\text { Migrant workers employed in Soutl } \\
\text { (in thousands) }\end{array}$ & 13.1 & $\ldots$ & $\ldots$ & $\ldots$ & $\ldots$ & $\ldots$ & \\
\hline
\end{tabular}

Sources: Central Statistics Office.

1/ Data for September of first year listed, except 1996/97 whose data are for March 1997.

2/ The increase in central government employment reflects the absorption of community, junior, and secondary school staff, the salaries of which were already being paid by the central government. 
Table 9. Botswana: Statutory Minimum Hourly Wage Rates, 1998-2004

\begin{tabular}{|c|c|c|c|c|c|c|c|}
\hline & 1998 & 1999 & 2000 & 2001 & 2002 & 2003 & 2004 \\
\hline & \multicolumn{7}{|c|}{ (In thebe) $1 /$} \\
\hline Manufacturing, service, and repair trades & 175 & 190 & 205 & 225 & 240 & 260 & 290 \\
\hline Building, construction, exploration, and quarrying & 175 & 190 & 205 & 225 & 240 & 260 & 290 \\
\hline Hotel, catering, and entertainment & 175 & 190 & 205 & 225 & 240 & 240 & 290 \\
\hline Garages, motor trade, and road transport & 175 & 190 & 205 & 225 & 225 & 260 & 255 \\
\hline Wholesale distributive trade & 165 & 180 & 205 & 225 & 240 & 260 & 290 \\
\hline Retail distributive trade & 155 & 170 & 185 & 205 & 215 & 230 & 290 \\
\hline Retail and wholesale nightwatchmen & 150 & 165 & 180 & 200 & 210 & 210 & 290 \\
\hline \multirow[t]{2}{*}{ Other nightwatchmen } & 150 & 165 & 180 & 200 & 210 & 220 & 245 \\
\hline & \multicolumn{7}{|c|}{ (Annual growth rate, in percent) } \\
\hline Manufacturing, service, and repair trades & 10.1 & 8.6 & 7.9 & 9.8 & 6.7 & 8.3 & 11.5 \\
\hline Building, construction, exploration, and quarrying & 10.1 & 8.6 & 7.9 & 9.8 & 6.7 & 8.3 & 11.5 \\
\hline Hotel, catering, and entertainment & 10.1 & 8.6 & 7.9 & 9.8 & 6.7 & 0.0 & 20.8 \\
\hline Garages, motor trade, and road transport & 10.1 & 8.6 & 7.9 & 9.8 & 0.0 & 15.6 & -1.9 \\
\hline Wholesale distributive trade & 8.6 & 9.1 & 13.9 & 9.8 & 6.7 & 8.3 & 11.5 \\
\hline Retail distributive trade & 8.4 & 9.7 & 8.8 & 10.8 & 4.9 & 7.0 & 26.1 \\
\hline Retail and wholesale nightwatchmen & 11.1 & 10.0 & 9.1 & 11.1 & 5.0 & 0.0 & 38.1 \\
\hline Other nightwatchmen & 11.1 & 10.0 & 9.1 & 11.1 & 5.0 & 4.8 & 11.4 \\
\hline
\end{tabular}

Source: Central Statistics Office.

$1 / 100$ thebe $=1$ pula. 
Table 10. Botswana: Average Monthly Cash Earnings by Sector, 1997-2002

\begin{tabular}{|c|c|c|c|c|c|c|}
\hline & 1997 & 1998 & 1999 & 2000 & 2001 & 2002 \\
\hline & \multicolumn{6}{|c|}{ (In pula) } \\
\hline Private and parastatal & 871 & 1,067 & 1,243 & 1327 & 1405 & 1903 \\
\hline Agriculture & 291 & 346 & 383 & 346 & 405 & 647 \\
\hline Mining and quarrying & 1,354 & 1,950 & 2,249 & 2,470 & 2,786 & 3,647 \\
\hline Manufacturing & 633 & 632 & 785 & 977 & 810 & 1084 \\
\hline Electricity and water & 1,857 & 2,043 & 3,166 & 3,330 & 3,194 & 4,811 \\
\hline Construction & 794 & 754 & 776 & 749 & 847 & 1257 \\
\hline Commerce & 623 & 867 & 950 & 1,004 & 1,121 & 1,567 \\
\hline Transport and communications & 1,255 & 1,725 & 2,318 & 2,553 & 2,631 & 3,759 \\
\hline Finance and business services & 1,348 & 1,593 & 2,059 & 2,156 & 2,339 & 3,708 \\
\hline Community and personal services & 912 & 1249 & 1,445 & 1,748 & 1,488 & 2,526 \\
\hline Education & 1,889 & 1,983 & 2,261 & 2,296 & 2,878 & 4,240 \\
\hline Local government & 964 & 1,190 & 1,496 & 1,698 & 1,829 & 2,466 \\
\hline Central government & 1,170 & 1,566 & 1,733 & 1,900 & 2,224 & 2,941 \\
\hline \multirow[t]{2}{*}{ Total } & 969 & 1,251 & 1,428 & 1,546 & 1,701 & 2,359 \\
\hline & \multicolumn{6}{|c|}{ (Annual percentage change) } \\
\hline Private and parastatal & 6.9 & 22.5 & 16.5 & 6.8 & 5.9 & 35.4 \\
\hline Agriculture & 9.0 & 18.9 & 10.7 & -9.7 & 17.1 & 59.8 \\
\hline Mining and quarrying & 9.4 & 44.0 & 15.3 & 9.8 & 12.8 & 30.9 \\
\hline Manufacturing & 2.6 & -0.2 & 24.2 & 24.5 & -17.1 & 33.8 \\
\hline Electricity and water & 35.4 & 10.0 & 55.0 & 5.2 & -4.1 & 50.6 \\
\hline Construction & 21.0 & -5.0 & 2.9 & -3.5 & 13.1 & 48.4 \\
\hline Commerce & 3.1 & 39.2 & 9.6 & 5.7 & 11.7 & 39.8 \\
\hline Transport and communications & 0.3 & 37.5 & 34.4 & 10.1 & 3.1 & 42.9 \\
\hline Finance and business services & 3.6 & 18.2 & 29.3 & 4.7 & 8.5 & 58.5 \\
\hline Community and personal services & 12.9 & 37.0 & 15.7 & 21.0 & -14.9 & 69.7 \\
\hline Education & 16.8 & 5.0 & 14.0 & 1.5 & 25.3 & 47.3 \\
\hline Local government & 1.8 & 23.4 & 25.7 & 13.5 & 7.7 & 34.8 \\
\hline Central government & 3.2 & 33.8 & 10.7 & 9.6 & 17.1 & 32.2 \\
\hline Total & 5.0 & 29.1 & 14.1 & 8.3 & 10.0 & 38.7 \\
\hline
\end{tabular}

Source: Central Statistics Office. 
Table 11. Botswana: Consumer Price Index of Tradables and Nontradables, 2000-04

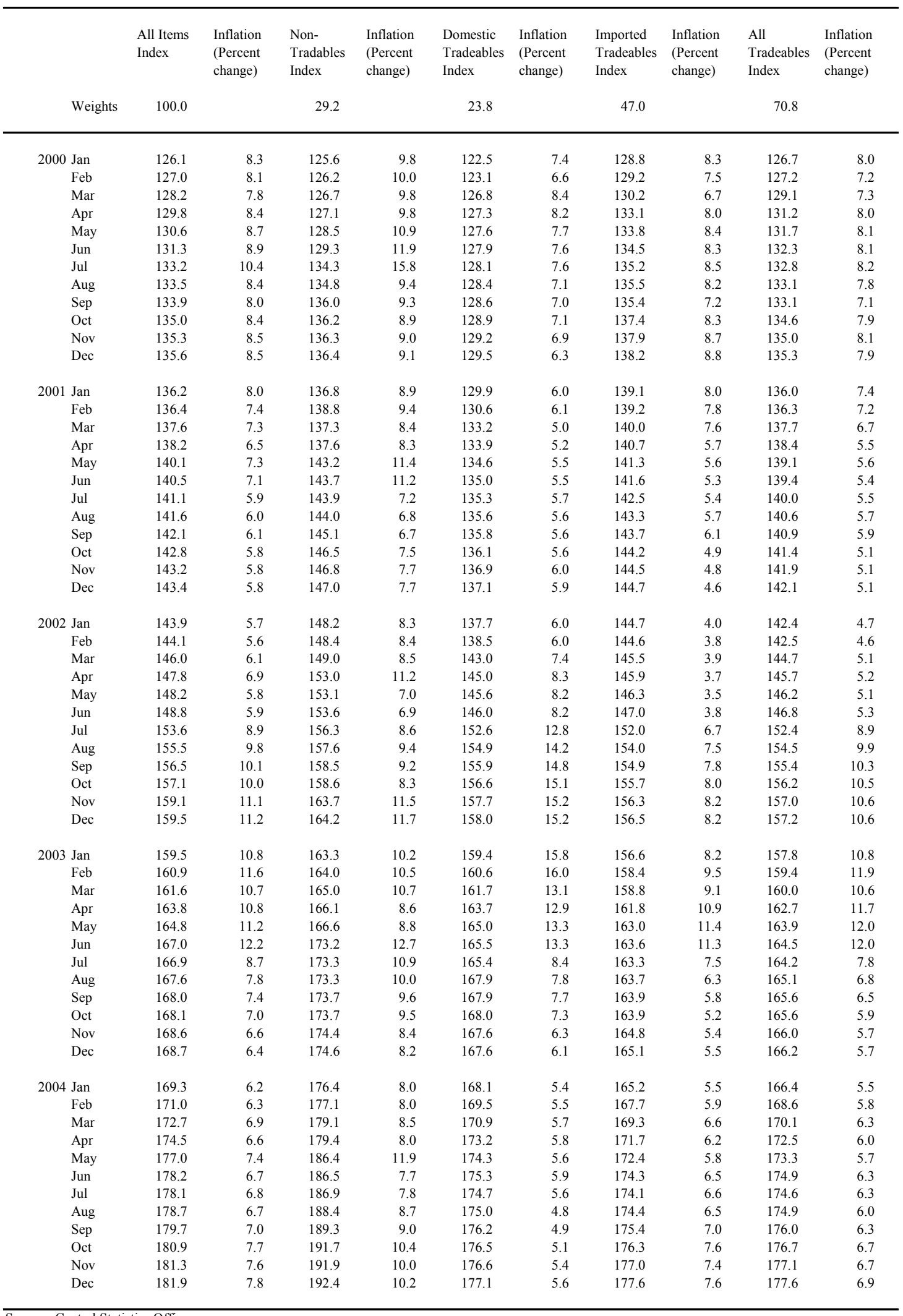


Table 12. Botswana: Cost of Living Index, 1991-2004 (Index; November 1996=100)

\begin{tabular}{|c|c|c|c|c|c|c|c|c|c|c|c|c|c|c|}
\hline & Food & $\begin{array}{l}\text { Alcohol } \\
\text { and } \\
\text { Tobacco }\end{array}$ & $\begin{array}{l}\text { Clothing } \\
\text { and } \\
\text { Footwear }\end{array}$ & Housing & $\begin{array}{l}\text { Fuel } \\
\text { and } \\
\text { Power }\end{array}$ & $\begin{array}{c}\text { Furniture } \\
\text { etc. }\end{array}$ & $\begin{array}{c}\text { H/hold } \\
\text { Operation }\end{array}$ & $\begin{array}{l}\text { Health, } \\
\text { Personal } \\
\text { Care }\end{array}$ & $\begin{array}{c}\text { Transport } \\
\text { etc. }\end{array}$ & Leisure & Education & Other & $\begin{array}{l}\text { All items } \\
\text { Index }\end{array}$ & $\begin{array}{l}\text { Annual } \\
\text { inflation }\end{array}$ \\
\hline Weights & 25.5 & 13.5 & 5.8 & 12.2 & 2.6 & 5.1 & 3.9 & 5.7 & 19.7 & 1.6 & 3.8 & 0.6 & 100.0 & $\%$ \\
\hline $1991 \mathrm{Dec}$ & 55.8 & 60.8 & 52.9 & 56.7 & 80.1 & 57.8 & 60.3 & 52.2 & 57.4 & 66.4 & 55.1 & 57.9 & 57.5 & 12.6 \\
\hline $1992 \mathrm{Dec}$ & 66.8 & 69.6 & 65.1 & 66.7 & 83.5 & 65.8 & 71.4 & 58.0 & 66.0 & 76.8 & 60.1 & 68.5 & 67.0 & 16.5 \\
\hline $1993 \mathrm{Dec}$ & 72.9 & 75.4 & 74.5 & 75.0 & 89.7 & 72.0 & 76.7 & 86.6 & 75.6 & 80.7 & 76.4 & 73.1 & 75.5 & 12.7 \\
\hline $1994 \mathrm{Dec}$ & 80.7 & 81.9 & 83.7 & 83.3 & 94.2 & 78.4 & 84.8 & 90.6 & 85.0 & 89.5 & 81.6 & 85.0 & 82.9 & 9.8 \\
\hline $1995 \mathrm{Dec}$ & 89.7 & 91.7 & 91.9 & 90.4 & 93.8 & 93.3 & 93.0 & 94.5 & 94.1 & 95.2 & 91.0 & 93.3 & 91.8 & 10.8 \\
\hline $1996 \mathrm{Dec}$ & 100.7 & 100.3 & 101.1 & 100.0 & 103.5 & 101.6 & 100.4 & 100.4 & 100.5 & 99.6 & 100.0 & 100.6 & 100.6 & 9.6 \\
\hline $1997 \mathrm{Dec}$ & 109.9 & 111.2 & 109.7 & 103.5 & 105.4 & 110.4 & 110.0 & 104.7 & 108.1 & 103.2 & 105.2 & 106.7 & 108.4 & 7.8 \\
\hline $1998 \mathrm{Dec}$ & 116.0 & 122.5 & 113.5 & 110.2 & 106.3 & 116.0 & 118.2 & 107.7 & 113.2 & 107.4 & 123.9 & 119.7 & 115.3 & 6.4 \\
\hline $1999 \mathrm{Dec}$ & 122.1 & 134.9 & 117.4 & 126.0 & 118.5 & 123.7 & 129.2 & 116.8 & 123.2 & 109.8 & 141.0 & 128.6 & 125.0 & 8.4 \\
\hline $2000 \mathrm{Dec}$ & 127.1 & 146.9 & 120.6 & 145.5 & 145.2 & 134.4 & 141.9 & 120.7 & 142.2 & 111.3 & 143.2 & 134.5 & 135.6 & 8.5 \\
\hline $2001 \mathrm{Dec}$ & 132.3 & 158.9 & 125.7 & 158.7 & 149.5 & 136.7 & 153.2 & 123.8 & 149.0 & 116.0 & 147.6 & 139.3 & 143.4 & 5.7 \\
\hline 2002 Jan & 132.9 & 159.2 & 125.9 & 159.0 & 149.5 & 137.5 & 153.8 & 124.1 & 148.2 & 117.5 & 158.2 & 139.4 & 143.9 & 5.7 \\
\hline Feb & 133.4 & 159.6 & 126.2 & 159.0 & 149.4 & 139.7 & 154.6 & 124.4 & 146.9 & 117.8 & 158.2 & 145.9 & 144.1 & 5.6 \\
\hline Mar & 135.0 & 165.1 & 126.5 & 160.3 & 149.4 & 139.5 & 155.1 & 124.4 & 148.5 & 118.1 & 158.5 & 151.8 & 146.0 & 6.1 \\
\hline Apr & 136.3 & 166.8 & 126.7 & 168.8 & 149.5 & 139.8 & 155.5 & 125.0 & 148.5 & 119.6 & 158.5 & 152.2 & 147.8 & 6.9 \\
\hline May & 136.9 & 167.0 & 127.0 & 169.0 & 149.6 & 141.5 & 155.5 & 125.2 & 148.6 & 120.2 & 158.5 & 152.4 & 148.2 & 5.8 \\
\hline Jun & 137.8 & 167.1 & 127.4 & 169.1 & 151.5 & 141.7 & 157.1 & 127.1 & 148.6 & 120.3 & 158.5 & 153.9 & 148.8 & 5.9 \\
\hline Jul & 143.9 & 174.9 & 128.4 & 171.0 & 160.6 & 142.9 & 159.7 & 129.4 & 153.7 & 121.6 & 164.0 & 158.4 & 153.6 & 8.8 \\
\hline Aug & 146.6 & 178.0 & 129.6 & 172.2 & 162.9 & 143.8 & 161.9 & 131.1 & 154.9 & 123.0 & 165.0 & 162.8 & 155.5 & 9.9 \\
\hline Sep & 147.9 & 179.0 & 130.2 & 173.8 & 163.2 & 144.1 & 164.5 & 131.8 & 154.9 & 123.6 & 164.8 & 162.9 & 156.5 & 10.1 \\
\hline Oct & 149.4 & 179.1 & 130.5 & 174.1 & 163.6 & 144.6 & 164.5 & 132.1 & 155.0 & 123.8 & 164.8 & 162.9 & 157.1 & 10.0 \\
\hline Nov & 150.7 & 179.3 & 130.8 & 174.3 & 163.2 & 144.8 & 165.1 & 132.6 & 164.7 & 124.1 & 164.8 & 171.7 & 159.1 & 11.1 \\
\hline Dec & 151.2 & 179.0 & 130.9 & 175.4 & 163.0 & 145.6 & 166.8 & 133.0 & 164.6 & 124.2 & 164.8 & 172.0 & 159.5 & 11.2 \\
\hline 2003 Jan & 152.3 & 179.4 & 130.9 & 175.9 & 161.6 & 146.2 & 167.0 & 137.6 & 164.0 & 125.0 & 173.1 & 176.2 & 160.4 & 11.5 \\
\hline Feb & 153.5 & 180.2 & 130.8 & 176.3 & 161.8 & 146.7 & 167.5 & 137.9 & 168.0 & 126.1 & 173.1 & 173.4 & 161.8 & 12.3 \\
\hline Mar & 154.7 & 181.2 & 131.1 & 176.8 & 161.3 & 147.0 & 168.2 & 137.8 & 168.0 & 126.1 & 177.9 & 173.5 & 162.5 & 11.3 \\
\hline Apr & 157.3 & 183.4 & 132.2 & 177.0 & 160.9 & 147.6 & 168.9 & 140.7 & 172.6 & 127.1 & 177.9 & 176.8 & 164.7 & 11.4 \\
\hline May & 158.7 & 184.7 & 132.9 & 178.2 & 162.3 & 147.7 & 170.4 & 141.0 & 173.4 & 127.0 & 177.7 & 176.9 & 165.7 & 11.8 \\
\hline Jun & 159.6 & 184.7 & 134.8 & 191.9 & 161.2 & 147.9 & 170.4 & 141.3 & 174.5 & 126.5 & 177.7 & 178.6 & 167.9 & 12.8 \\
\hline Jul & 159.6 & 184.7 & 134.9 & 192.2 & 158.8 & 148.3 & 170.7 & 141.5 & 173.3 & 126.5 & 177.7 & 179.1 & 167.8 & 9.2 \\
\hline Aug & 160.1 & 187.1 & 134.8 & 192.2 & 158.9 & 148.9 & 171.7 & 141.6 & 173.4 & 126.4 & 177.7 & 180.0 & 168.5 & 8.4 \\
\hline Sep & 160.3 & 188.3 & 135.2 & 193.0 & 158.7 & 149.4 & 173.0 & 141.7 & 166.9 & 126.5 & 177.7 & 178.8 & 167.9 & 7.3 \\
\hline Oct & 159.7 & 188.7 & 135.4 & 193.0 & 158.4 & 150.0 & 172.9 & 141.6 & 168.3 & 126.5 & 177.7 & 178.8 & 168.1 & 7.0 \\
\hline Nov & 159.8 & 188.5 & 135.6 & 194.0 & 159.7 & 150.4 & 173.2 & 142.0 & 170.2 & 126.6 & 177.7 & 178.9 & 168.6 & 6.0 \\
\hline Dec & 160.1 & 188.2 & 135.8 & 194.7 & 159.8 & 150.7 & 174.2 & 142.1 & 170.2 & 126.7 & 177.7 & 179.1 & 168.7 & 5.8 \\
\hline 2004 Jan & 160.8 & 188.0 & 136.2 & 195.0 & 158.6 & 150.9 & 173.2 & 142.3 & 170.2 & 125.8 & 192.0 & 179.2 & 169.3 & 5.5 \\
\hline Feb & 161.4 & 190.3 & 136.7 & 195.6 & 158.6 & 151.4 & 173.9 & 143.1 & 175.7 & 127.5 & 192.0 & 180.0 & 171.0 & 5.7 \\
\hline Mar & 163.1 & 192.7 & 136.1 & 196.8 & 162.7 & 150.5 & 173.4 & 145.0 & 178.9 & 127.1 & 192.0 & 184.5 & 172.7 & 6.3 \\
\hline Apr & 165.5 & 195.9 & 136.4 & 197.5 & 166.3 & 151.0 & 175.7 & 146.0 & 181.1 & 128.7 & 192.1 & 184.7 & 174.5 & 6.0 \\
\hline May & 166.1 & 199.3 & 135.9 & 212.2 & 167.0 & 150.8 & 174.5 & 145.7 & 181.3 & 128.4 & 192.3 & 184.2 & 177.0 & 6.8 \\
\hline Jun & 167.0 & 200.4 & 136.1 & 211.4 & 172.2 & 151.4 & 176.8 & 146.9 & 184.6 & 128.6 & 192.3 & 185.1 & 178.2 & 6.1 \\
\hline Jul & 165.9 & 200.0 & 136.8 & 211.8 & 173.4 & 151.7 & 176.6 & 147.3 & 184.7 & 129.2 & 192.3 & 191.4 & 178.1 & 6.1 \\
\hline Aug & 166.3 & 200.1 & 137.0 & 212.0 & 173.4 & 152.3 & 177.7 & 147.5 & 188.7 & 129.5 & 192.3 & 191.2 & 178.7 & 6.1 \\
\hline Sep & 166.6 & 201.8 & 136.7 & 213.1 & 176.0 & 153.5 & 181.3 & 147.8 & 190.6 & 128.5 & 192.3 & 192.2 & 179.7 & 7.0 \\
\hline Oct & 167.3 & 203.5 & 137.1 & 214.9 & 175.8 & 153.5 & 181.9 & 147.6 & 194.0 & 129.0 & 192.3 & 192.6 & 180.9 & 7.7 \\
\hline Nov & 167.4 & 204.8 & 136.8 & 215.7 & 177.2 & 152.7 & 182.6 & 148.0 & 194.2 & 130.3 & 192.3 & 193.5 & 181.3 & 7.6 \\
\hline Dec & 168.0 & 205.4 & 137.4 & 216.4 & 179.1 & 152.7 & 183.6 & 149.0 & 194.3 & 130.7 & 192.3 & 194.1 & 181.9 & 7.8 \\
\hline
\end{tabular}


Table 13. Botswana: Central Government Operations, 1998/99-2005/06 1/

\begin{tabular}{|c|c|c|c|c|c|c|c|c|}
\hline & $1998 / 99$ & $1999 / 00$ & $2000 / 01$ & $2001 / 02$ & $2002 / 03$ & $2003 / 04$ & $\begin{array}{r}2004 / 05 \\
\text { Est. }\end{array}$ & $\begin{array}{r}2005 / 06 \\
\text { Budge }\end{array}$ \\
\hline & \multicolumn{8}{|c|}{ (In millions of pula) } \\
\hline Total revenue and grants & 7,617 & 11,910 & 14,050 & 12,601 & 14,240 & 15,348 & 16,961 & 20,179 \\
\hline Total revenue & 7,479 & 11,784 & 13,986 & 12,542 & 14,155 & 15,287 & 16,761 & 19,959 \\
\hline Tax revenue & 5,640 & 9,938 & 12,078 & 10,582 & 12,259 & 13,376 & 15,192 & 18,157 \\
\hline Mineral revenue & 3,187 & 6,687 & 8,368 & 6,996 & 7,503 & 7,393 & 7,713 & 9,926 \\
\hline Trade and excise taxes $2 /$ & 1,261 & 1,931 & 2,188 & 1,732 & 1,569 & 2,246 & 3,292 & 3,407 \\
\hline General sales tax & 401 & 484 & 524 & 520 & 1,255 & 1,573 & 2,000 & 2,100 \\
\hline Other & 791 & 836 & 998 & 1,334 & 1,933 & 2,165 & 2,187 & 2,724 \\
\hline Nontax revenue & 1,840 & 1,846 & 1,908 & 1,960 & 1,896 & 1,911 & 1,570 & 1,802 \\
\hline Interest & 209 & 166 & 205 & 189 & 227 & 208 & 165 & 75 \\
\hline Property income & 1,253 & 1,232 & 1,195 & 1,170 & 1,064 & 969 & 572 & 462 \\
\hline Other & 378 & 448 & 508 & 601 & 605 & 733 & 833 & 1,265 \\
\hline Grants & 138 & 126 & 65 & 59 & 84 & 61 & 200 & 220 \\
\hline Total expenditure and lending & 9,065 & 10,427 & 11,536 & 13,671 & 15,710 & 16,275 & 18,720 & 20,454 \\
\hline Current expenditure & 6,265 & 7,048 & 8,383 & 9,935 & 11,581 & 12,934 & 14,625 & 15,720 \\
\hline Wages and salaries & 2,153 & 2,419 & 2,743 & 3,446 & 3,947 & 4,142 & 4,776 & 5,436 \\
\hline Interest & 93 & 93 & 83 & 94 & 81 & 193 & 353 & 305 \\
\hline Other & 4,019 & 4,537 & 5,557 & 6,394 & 7,553 & 8,600 & 9,496 & 9,979 \\
\hline Capital expenditure & 2,935 & 3,451 & 3,135 & 3,698 & 4,200 & 4,256 & 4,327 & 4,858 \\
\hline Net lending & -134 & -71 & 19 & 38 & -71 & -916 & -232 & -124 \\
\hline Primary balance (deficit -) & $-1,356$ & 1,575 & 2,597 & -975 & $-1,389$ & -734 & $-1,405$ & 30 \\
\hline Overall balance (deficit -) & $-1,449$ & 1,483 & 2,514 & $-1,069$ & $-1,471$ & -927 & $-1,758$ & -275 \\
\hline Financing 3/ & 1,449 & $-1,483$ & $-2,514$ & $-1,069$ & 1,932 & 927 & 1,758 & 273 \\
\hline Foreign (net) & 28 & -35 & -177 & -184 & -250 & -113 & -90 & -906 \\
\hline Drawing & 194 & 130 & 34 & 60 & 24 & 62 & 97 & 97 \\
\hline Amortization & -166 & -169 & -211 & -244 & -274 & -174 & -187 & $-1,003$ \\
\hline \multirow[t]{2}{*}{ Domestic } & 1,421 & $-1,448$ & $-2,337$ & 1,254 & 2,182 & 1,040 & 1,848 & 1,179 \\
\hline & \multicolumn{8}{|c|}{ (In percent of GDP) } \\
\hline Total revenue and grants & 36.0 & 49.4 & 50.7 & 40.5 & 40.1 & 39.3 & 39.2 & 42.3 \\
\hline Total revenue & 35.3 & 48.9 & 50.5 & 40.3 & 39.9 & 39.1 & 38.8 & 41.8 \\
\hline Tax revenue & 26.6 & 41.3 & 43.6 & 34.0 & 34.5 & 34.2 & 35.2 & 38.0 \\
\hline Mineral revenue & 15.0 & 27.8 & 30.2 & 22.5 & 21.1 & 18.9 & 17.8 & 20.8 \\
\hline Trade and excise taxes $2 /$ & 6.0 & 8.0 & 7.9 & 5.6 & 4.4 & 5.7 & 7.6 & 7.1 \\
\hline General sales tax & 1.9 & 2.0 & 1.9 & 1.7 & 3.5 & 4.0 & 4.6 & 4.4 \\
\hline Other & 3.7 & 3.5 & 3.6 & 4.3 & 5.4 & 5.5 & 5.1 & 5.7 \\
\hline Nontax revenue & 8.7 & 7.7 & 6.9 & 6.3 & 5.3 & 4.9 & 3.6 & 3.8 \\
\hline Interest & 1.0 & 0.7 & 0.7 & 0.6 & 0.6 & 0.5 & 0.4 & 0.2 \\
\hline Property income & 5.9 & 5.1 & 4.3 & 3.8 & 3.0 & 2.5 & 1.3 & 1.0 \\
\hline Other & 1.8 & 1.9 & 1.8 & 1.9 & 1.7 & 1.9 & 1.9 & 2.6 \\
\hline Grants & 0.7 & 0.5 & 0.2 & 0.2 & 0.2 & 0.2 & 0.5 & 0.5 \\
\hline Total expenditure and lending & 42.8 & 43.3 & 41.6 & 44.0 & 44.2 & 41.6 & 43.3 & 42.8 \\
\hline Current expenditure & 29.6 & 29.3 & 30.2 & 31.9 & 32.6 & 33.1 & 33.8 & 32.9 \\
\hline Wages and salaries & 10.2 & 10.0 & 9.9 & 11.1 & 11.1 & 10.6 & 11.1 & 11.4 \\
\hline Interest & 0.4 & 0.4 & 0.3 & 0.3 & 0.2 & 0.5 & 0.8 & 0.6 \\
\hline Other & 19.0 & 18.8 & 20.1 & 20.6 & 21.3 & 22.0 & 22.0 & 20.9 \\
\hline Capital expenditure & 13.9 & 14.3 & 11.3 & 11.9 & 11.8 & 10.9 & 10.0 & 10.2 \\
\hline Net lending & -0.6 & -0.3 & 0.1 & 0.1 & -0.2 & -2.3 & -0.5 & -0.3 \\
\hline Primary balance (deficit -) & -6.4 & 6.5 & 9.4 & -3.1 & -3.9 & -1.9 & -3.3 & 0.1 \\
\hline Overall balance (deficit -) & -6.8 & 6.2 & 9.1 & -3.4 & -4.1 & -2.4 & -4.1 & -0.6 \\
\hline \multicolumn{9}{|l|}{ Memorandum item: } \\
\hline GDP (fiscal year; in millions of pula) & 21,183 & 24,088 & 27,713 & 31,101 & 35,517 & 39,089 & 43,216 & 47,743 \\
\hline
\end{tabular}

Sources: Ministry of Finance and Development Planning; and IMF staff estimates.

1/ Fiscal year beginning April 1

2/ Trade and excise taxes are received from the revenue pool of the Southern African Customs Union.

3/ The figures for 2002/03 include issuance of government bonds aimed at developing the domestic capital market. 
Table 14. Botswana: Components of Central Government Revenue, 1998/99-2005/06 1/ (In millions of pula)

\begin{tabular}{|c|c|c|c|c|c|c|c|c|}
\hline & 1998/99 & $1999 / 00$ & $2000 / 01$ & $2001 / 02$ & $2002 / 03$ & $2003 / 04$ & $\begin{array}{r}2004 / 05 \\
\text { Est. }\end{array}$ & $\begin{array}{r}2005 / 06 \\
\text { Budget } \\
\end{array}$ \\
\hline Tax revenue & 5,640 & 9,938 & 12,078 & 10,582 & 12,259 & 13,376 & 15,192 & 18,157 \\
\hline Mineral revenue & 3,187 & 6,687 & 8,368 & 6,996 & 7,503 & 7,393 & 7,713 & 9,926 \\
\hline Trade and excise taxes $2 /$ & 1,261 & 1,931 & 2,188 & 1,732 & 1,569 & 2,246 & 3,292 & 3,407 \\
\hline General sales tax & 401 & 484 & 524 & 520 & 1,255 & 1,573 & 2,000 & 2,100 \\
\hline Nonmineral income tax & 739 & 780 & 925 & 1,249 & 1,840 & 2,079 & 2,069 & 2,600 \\
\hline Export duties & 0 & 0 & 0 & $\ldots$ & $\ldots$ & $\ldots$ & $\ldots$ & $\ldots$ \\
\hline Taxes on property & 11 & 12 & 16 & 16 & 18 & 12 & 22 & 18 \\
\hline Motor vehicle tax & 26 & 27 & 40 & 51 & 55 & 62 & 75 & 75 \\
\hline Business and professional licenses & 12 & 14 & 14 & 16 & 18 & 9 & 21 & 19 \\
\hline Airport tax & 3 & 3 & 2 & 3 & 2 & 3 & $\ldots$ & 12 \\
\hline Nontax revenue & 1,840 & 1,846 & 1,908 & 1,960 & 1,896 & 1,911 & 1,570 & 1,802 \\
\hline Interest & 209 & 166 & 205 & 189 & 227 & 208 & 165 & 75 \\
\hline Property income & 1,253 & 1,232 & 1,195 & 1,170 & 1,064 & 969 & 572 & 462 \\
\hline Fees, charges, and reimbursements & 378 & 448 & 508 & 601 & 605 & 733 & 833 & 1,265 \\
\hline Grants & 138 & 126 & 65 & 59 & 84 & 61 & 200 & 220 \\
\hline Recurrent & 1 & 0 & $\ldots$ & $\ldots$ & 0 & 0 & $\ldots$ & $\ldots$ \\
\hline Development & 136 & 126 & 65 & 59 & 84 & 61 & 200 & 220 \\
\hline Total revenue and grants & 7,617 & 11,910 & 14,050 & 12,601 & 14,239 & 15,348 & 16,962 & 20,179 \\
\hline
\end{tabular}

Source: Ministry of Finance and Development Planning; and IMF staff estimates.

1/ Fiscal year beginning April 1.

2/ Trade and excise taxes are received from the revenue pool of the Southern African Customs Union. 
Table 15. Botswana: Economic Classification of Central Government Expenditure, 1998/99-2005/06 1/

\begin{tabular}{|c|c|c|c|c|c|c|c|c|}
\hline & $1998 / 99$ & $1999 / 00$ & $2000 / 01$ & $2001 / 02$ & $2002 / 03$ & $2003 / 04$ & $\begin{array}{r}2004 / 05 \\
\text { Est. }\end{array}$ & $\begin{array}{r}2005 / 06 \\
\text { Budget } \\
\end{array}$ \\
\hline & \multicolumn{8}{|c|}{ (In millions of pula) } \\
\hline Total expenditure and net lending & 9,065 & 10,427 & 11,536 & 13,671 & 15,710 & 16,275 & 18,720 & 20,454 \\
\hline Current expenditure & 6,265 & 7,048 & 8,383 & 9,935 & 11,581 & 12,934 & 14,625 & 15,720 \\
\hline Expenditure on goods and services & 6,173 & 6,955 & 8,300 & 9,841 & 11,500 & 12,742 & 14,272 & 15,415 \\
\hline Wages and salaries & 2,153 & 2,419 & 2,743 & 3,446 & 3,947 & 4,142 & 4,776 & 5,436 \\
\hline Other purchases of goods and services $2 /$ & 4,019 & 4,537 & 5,557 & 6,394 & 7,553 & 8,600 & 9,496 & 9,979 \\
\hline Interest payments & 93 & 93 & 83 & 94 & 81 & 193 & 353 & 305 \\
\hline Capital expenditure & 2,935 & 3,451 & 3,135 & 3,698 & 4,200 & 4,256 & 4,327 & 4,858 \\
\hline Net lending & -134 & -71 & 19 & 38 & -71 & -916 & -232 & -124 \\
\hline Gross lending & 42 & 110 & 120 & 150 & 10 & 0 & 0 & 0 \\
\hline \multirow[t]{2}{*}{ Repayment } & -176 & -181 & -101 & -112 & -81 & -916 & -232 & -124 \\
\hline & \multicolumn{8}{|c|}{ (Percent share of total expenditure) } \\
\hline Total expenditure and net lending & 100.0 & 100.0 & 100.0 & 100.0 & 100.0 & 100.0 & 100.0 & 100.0 \\
\hline Current expenditure & 69.1 & 67.6 & 72.7 & 72.7 & 73.7 & 79.5 & 78.1 & 76.9 \\
\hline Expenditure on goods and services & 68.1 & 66.7 & 71.9 & 72.0 & 73.2 & 78.3 & 76.2 & 75.4 \\
\hline Wages and salaries & 23.8 & 23.2 & 23.8 & 25.2 & 25.1 & 25.4 & 25.5 & 26.6 \\
\hline Other purchases of goods and services $2 /$ & 44.3 & 43.5 & 48.2 & 46.8 & 48.1 & 52.8 & 50.7 & 48.8 \\
\hline Interest payments & 1.0 & 0.9 & 0.7 & 0.7 & 0.5 & 1.2 & 1.9 & 1.5 \\
\hline Capital expenditure & 32.4 & 33.1 & 27.2 & 27.1 & 26.7 & 26.2 & 23.1 & 23.8 \\
\hline Net lending & -1.5 & -0.7 & 0.2 & 0.3 & -0.5 & -5.6 & -1.2 & -0.6 \\
\hline Gross lending & 0.5 & 1.1 & 1.0 & 1.1 & 0.1 & 0.0 & 0.0 & 0.0 \\
\hline Repayment & -1.9 & -1.7 & -0.9 & -0.8 & -0.5 & -5.6 & -1.2 & -0.6 \\
\hline
\end{tabular}

Sources: Ministry of Finance and Development Planning; and IMF staff estimates.

1/ Fiscal year beginning April 1.

2/ Includes transfers. 
Table 16. Botswana: Functional Classification of Central Government Expenditure, 1998/99-2005/06 1/

\begin{tabular}{|c|c|c|c|c|c|c|c|c|}
\hline & 1998/99 & 1999/00 & $2000 / 01$ & $2001 / 02$ & $2002 / 03$ & 2003/04 & $\begin{array}{r}2004 / 05 \\
\text { Est. }\end{array}$ & $\begin{array}{r}2005 / 06 \\
\text { Budget } \\
\end{array}$ \\
\hline & \multicolumn{8}{|c|}{ (In millions of pula) } \\
\hline General public services & 1,674 & 2,146 & 2,298 & 2,400 & 2,968 & 3,265 & 3,624 & 4,025 \\
\hline General administration & 1,230 & 1,534 & 1,612 & 1,771 & 2,195 & 2,414 & 2,680 & 3,093 \\
\hline Public order and safety & 444 & 612 & 686 & 629 & 773 & 850 & 944 & 931 \\
\hline Defense & 812 & 775 & 998 & 1,305 & 1,451 & 1,596 & 1,772 & 1,545 \\
\hline Social services & 4,005 & 4,508 & 5,033 & 5,876 & 6,889 & 8,486 & 9,122 & 9,800 \\
\hline Education & 2,276 & 2,458 & 2,872 & 3,409 & 3,597 & 4,497 & 4,711 & 5,122 \\
\hline Health & 468 & 543 & 630 & 803 & 1,103 & 1,447 & 2,041 & 2,501 \\
\hline Food and social welfare programs & 321 & 372 & 424 & 463 & 350 & 518 & 123 & 83 \\
\hline Housing and urban and regional development & 670 & 734 & 762 & 827 & 1,218 & 1,340 & 1,487 & 1,368 \\
\hline Other community and social services & 270 & 401 & 345 & 373 & 621 & 684 & 759 & $\begin{array}{r}725 \\
9.074\end{array}$ \\
\hline Economic services & 1,800 & 2,099 & 2,042 & 2,751 & 3,134 & 3,291 & 3,456 & 3,388 \\
\hline Agriculture, forestry, and fishing & 440 & 451 & 482 & 570 & 688 & 757 & 841 & 673 \\
\hline Mining & 201 & 124 & 75 & 394 & 101 & 112 & 124 & 106 \\
\hline Roads, other transport, and communications & 451 & 646 & 576 & 668 & 994 & 1,093 & 1,213 & 1,098 \\
\hline Electricity and water supply & 391 & 535 & 580 & 695 & 742 & 816 & 906 & 594 \\
\hline Commerce, industry, and other & 317 & 343 & 330 & 424 & 609 & 757 & 802 & 917 \\
\hline Unallocated expenditure and adjustments & 775 & 899 & 1,166 & 1,338 & 1,339 & 1,805 & 2,505 & 3,242 \\
\hline \multirow[t]{2}{*}{ Total } & 9,065 & 10,427 & 11,536 & 13,671 & 15,710 & 16,275 & 18,720 & 20,454 \\
\hline & \multicolumn{8}{|c|}{ (Percent share of total expenditure) } \\
\hline General public services & 18.5 & 20.6 & 19.9 & 17.6 & 18.9 & 20.1 & 19.4 & 19.7 \\
\hline General administration & 13.6 & 14.7 & 14.0 & 13.0 & 14.0 & 14.8 & 14.3 & 15.1 \\
\hline Public order and safety & 4.9 & 5.9 & 5.9 & 4.6 & 4.9 & 5.2 & 5.0 & 4.6 \\
\hline Defense & 9.0 & 7.4 & 8.7 & 9.5 & 9.2 & 9.8 & 9.5 & 7.6 \\
\hline Social services & 44.2 & 43.2 & 43.6 & 43.0 & 43.9 & 52.1 & 48.7 & 47.9 \\
\hline Education & 25.1 & 23.6 & 24.9 & 24.9 & 22.9 & 27.6 & 25.2 & 25.0 \\
\hline Health & 5.2 & 5.2 & 5.5 & 5.9 & 7.0 & 8.9 & 10.9 & 12.2 \\
\hline Food and social welfare programs & 3.5 & 3.6 & 3.7 & 3.4 & 2.2 & 3.2 & 0.7 & 0.4 \\
\hline Housing and urban and regional development & 7.4 & 7.0 & 6.6 & 6.1 & 7.8 & 8.2 & 7.9 & 6.7 \\
\hline Other community and social services & 3.0 & 3.8 & 3.0 & 2.7 & 4.0 & 4.2 & 4.1 & 3.5 \\
\hline Economic services & 19.9 & 20.1 & 17.7 & 20.1 & 19.9 & 19.1 & 18.2 & 16.6 \\
\hline Agriculture, forestry, and fishing & 4.9 & 4.3 & 4.2 & 4.2 & 4.4 & 4.7 & 4.5 & 3.3 \\
\hline Mining & 2.2 & 1.2 & 0.6 & 2.9 & 0.6 & 0.7 & 0.7 & 0.5 \\
\hline Roads, other transport, and communications & 5.0 & 6.2 & 5.0 & 4.9 & 6.3 & 6.7 & 6.5 & 5.4 \\
\hline Electricity and water supply & 4.3 & 5.1 & 5.0 & 5.1 & 4.7 & 5.0 & 4.8 & 2.9 \\
\hline Commerce, industry, and other & 3.5 & 3.3 & 2.9 & 3.1 & 3.9 & 3.7 & 3.5 & 4.5 \\
\hline Unallocated expenditure and adjustments & 8.5 & 8.6 & 10.1 & 9.8 & 8.5 & 10.5 & 13.2 & 15.9 \\
\hline Total & 100.0 & 100.0 & 100.0 & 100.0 & 100.0 & 100.0 & 100.0 & 100.0 \\
\hline
\end{tabular}

1/ Fiscal year beginning April 1 . 
Table 17. Botswana: Summary of Operations of Nonfinancial Public Enterprises, 1998-2003 1/

\begin{tabular}{|c|c|c|c|c|c|c|}
\hline & 1998 & 1999 & 2000 & 2001 & 2002 & 2003 \\
\hline & \multicolumn{6}{|c|}{ (In millions of pula) } \\
\hline Operating revenue & 1,481 & 1,557 & 1,611 & 2,300 & 2,342 & 2,425 \\
\hline Net profit/loss & 316 & 379 & 433 & 386 & 272 & 311 \\
\hline Long-term debt outstanding & 1,813 & 2,208 & 2,094 & 2,102 & 2,013 & 1,732 \\
\hline Equity & 52,552 & 3,097 & 4,393 & 4,991 & 5,321 & 4,946 \\
\hline Capital employed & 4,825 & 5,915 & 6,105 & 7,449 & 7,783 & 6,950 \\
\hline \multirow[t]{2}{*}{ Fixed assets } & 3,554 & 5,269 & 5,531 & 5,921 & 6,087 & 5,552 \\
\hline & \multicolumn{6}{|c|}{ (In percent, period average, unless otherwise indicated) } \\
\hline Return on capital employed & 12.0 & 7.1 & 3.3 & 12.8 & 3.1 & -7.0 \\
\hline Return on equity & 9.2 & 8.9 & 3.5 & 14.1 & 3.8 & -8.7 \\
\hline Net profit-to-sales ratio & 17.0 & 20.7 & 17.6 & 23.6 & 11.8 & 6.8 \\
\hline Debt-to-equity ratio & 29.1 & 38.2 & 35.4 & 57.3 & 56.7 & 53.1 \\
\hline \multicolumn{7}{|l|}{ Memorandum item: } \\
\hline Operating revenue (in percent of GDP) & 7.3 & 7.2 & 6.5 & 8.0 & 7.3 & 6.6 \\
\hline
\end{tabular}

Source: Bank of Botswana.

1/ Includes the Botswana Agricultural Marketing Board, the Botswana Livestock Development Corporation, the Botswana Housing Corporation, the Botswana Meat Commission, the Botswana Power Corporation, the Botswana Telecommunications Corporation, and the Botswana Water Utilities Corporation. 
Table 18. Botswana: Monetary Survey, 1998-2004

\begin{tabular}{|c|c|c|c|c|c|c|c|}
\hline & 1998 & 1999 & 2000 & 2001 & 2002 & 2003 & 2004 \\
\hline & \multicolumn{7}{|c|}{ (In millions of pula; end of period) } \\
\hline Net foreign assets & 27,747 & 30,051 & 35,110 & 43,075 & 31,247 & 25,238 & 25,519 \\
\hline Bank of Botswana & 26,502 & 28,867 & 33,900 & 41,211 & 29,984 & 23,887 & 24,368 \\
\hline Commercial banks & 1,245 & 1,185 & 1,210 & 1,863 & 1,263 & 1,351 & 1,152 \\
\hline Net domestic credit & $-16,128$ & $-15,962$ & $-19,205$ & $-22,320$ & $-9,870$ & $-3,460$ & $-1,264$ \\
\hline Net claims on the government & $-19,087$ & $-20,137$ & $-24,130$ & $-27,778$ & $-16,491$ & $-10,662$ & $-9,705$ \\
\hline Bank of Botswana & $-19,072$ & $-20,086$ & $-24,026$ & $-27,719$ & $-16,433$ & $-10,514$ & $-9,272$ \\
\hline Commercial banks & -15 & -51 & -104 & -60 & -58 & -148 & -433 \\
\hline Claims on nongovernment & 2,958 & 4,176 & 4,926 & 5,459 & 6,621 & 7,202 & 8,441 \\
\hline Claims on parastatals & 267 & 528 & 458 & 480 & 462 & 381 & 433 \\
\hline Claims on the private sector & 2,692 & 3,648 & 4,468 & 4,979 & 6,159 & 6,821 & 8,007 \\
\hline Other items (net) & $-5,898$ & $-6,861$ & $-8,577$ & $-11,138$ & $-11,870$ & $-10,801$ & $-11,524$ \\
\hline \multicolumn{8}{|l|}{ Of which } \\
\hline Valuation adjustment $1 /$ & $-3,719$ & $-8,800$ & $-11,304$ & $-15,973$ & $-11,144$ & $-8,131$ & 869 \\
\hline Money plus quasi-money & 5,722 & 7,228 & 7,328 & 9,617 & 9,508 & 10,977 & 12,731 \\
\hline Money & 1,513 & 1,775 & 1,897 & 2,351 & 2,524 & 2,822 & 3,626 \\
\hline Quasi money 2/ & 4,209 & 5,454 & 5,432 & 7,266 & 6,984 & 8,155 & 9,105 \\
\hline \multicolumn{8}{|l|}{ Memorandum items: } \\
\hline Broad money (M3) & 8,968 & 11,458 & 11,041 & 14,764 & 17,171 & 19,717 & 22,380 \\
\hline \multicolumn{8}{|l|}{ Of which } \\
\hline Bank of Botswana certificates & 988 & 1,421 & 1,229 & 1,303 & 2,425 & 2,780 & 3,023 \\
\hline Broad money (M4) & 9,907 & 12,554 & 12,227 & 16,743 & 18,709 & 21,234 & 24,525 \\
\hline \multicolumn{8}{|l|}{ Of which } \\
\hline \multirow[t]{2}{*}{ Foreign currency accounts } & 939 & 1,096 & 1,186 & 1,978 & 1,538 & 1,518 & 2,145 \\
\hline & \multicolumn{7}{|c|}{ (Twelve-month percentage change) } \\
\hline Net foreign assets 3 / & 24.3 & 8.3 & 16.8 & 22.7 & -27.5 & -19.2 & 1.1 \\
\hline Net domestic credit & -19.5 & 1.0 & -20.3 & -16.2 & 55.8 & 64.9 & 63.5 \\
\hline \multicolumn{8}{|l|}{ Of which } \\
\hline Claims on the government (net) & -24.0 & -5.5 & -19.8 & -15.1 & 40.6 & 35.3 & 9.0 \\
\hline Claims on the private sector & 46.6 & 35.5 & 22.5 & 11.4 & 23.7 & 10.7 & 17.4 \\
\hline Money plus quasi-money & 39.4 & 26.3 & 1.4 & 31.2 & -1.1 & 15.5 & 16.0 \\
\hline
\end{tabular}

Source: Bank of Botswana.

1/ Equivalent to the revaluation profit (loss) for the year reported on the books of the Bank of Botswana.

$2 /$ Includes private deposits at the Bank of Botswana but excludes holdings of Bank of Botswana certificates.

3/ Excludes the effect of foreign assets valuation adjustments. 
Table 19. Botswana: Summary Accounts of Bank of Botswana, 1998-2004 1/ (In millions of pula; end of period)

\begin{tabular}{|c|c|c|c|c|c|c|c|}
\hline & 1998 & 1999 & 2000 & 2001 & 2002 & 2003 & 2004 \\
\hline Foreign assets & 26,502 & 28,867 & 33,900 & 41,211 & 29,984 & 23,887 & 24,368 \\
\hline Pula fund & 23,562 & 24,454 & 28,712 & 32,176 & 25,524 & 19,246 & 20,013 \\
\hline Liquidity portfolio & 2,545 & 4,075 & 4,833 & 8,534 & 3,985 & 4,055 & 3,827 \\
\hline Matched assets/liability portfolio & 19 & 16 & 23 & 30 & 56 & 166 & 163 \\
\hline Fund accounts & 377 & 322 & 333 & 472 & 419 & 421 & 365 \\
\hline Holding of SDRs & 203 & 178 & 209 & 277 & 243 & 221 & 229 \\
\hline Reserve position & 173 & 144 & 124 & 195 & 176 & 197 & 134 \\
\hline Valuation Adjustment & 0 & 0 & 0 & 1 & 1 & 3 & 2 \\
\hline Loans and advances to financial institutions & 0 & 0 & 0 & 0 & 0 & 0 & 0 \\
\hline Fixed assets & 108 & 122 & 131 & 129 & 127 & 127 & 130 \\
\hline Other assets & 2 & 2 & 2 & 0 & -2 & -5 & -5 \\
\hline Assets $=$ liabilities & 26,612 & 28,991 & 34,034 & 41,341 & 30,109 & 24,009 & 24,493 \\
\hline Reserve money & 707 & 808 & 857 & 970 & 1,050 & 1,338 & 1,262 \\
\hline Currency in circulation & 498 & 607 & 606 & 701 & 759 & 818 & 911 \\
\hline Currency outside banks & 353 & 404 & 427 & 481 & 470 & 533 & 637 \\
\hline Pula currency in banks & 145 & 203 & 180 & 220 & 289 & 285 & 274 \\
\hline Bankers' deposits & 210 & 201 & 251 & 268 & 291 & 520 & 351 \\
\hline Private sector time deposits & 26 & 172 & 183 & 184 & 286 & 231 & 852 \\
\hline Bank of Botswana certificates outstanding & 3,246 & 4,230 & 3,712 & 5,148 & 7,663 & 8,739 & 9,649 \\
\hline Bankers & 2,258 & 2,809 & 2,484 & 3,845 & 5,612 & 6,583 & 6,453 \\
\hline Others & 988 & 1,421 & 1,229 & 1,303 & 2,425 & 2,780 & 3,023 \\
\hline Government deposits & 19,072 & 20,086 & 24,026 & 27,719 & 16,433 & 10,514 & 9,272 \\
\hline Capital and reserves & 3,202 & 3,387 & 4,354 & 6,630 & 4,075 & 2,730 & 3,026 \\
\hline Paid-up capital & 25 & 25 & 25 & 25 & 25 & 25 & 25 \\
\hline General reserve & 1,600 & 1,600 & 1,600 & 1,600 & 1,600 & 1,600 & 1,600 \\
\hline Revaluation reserve & 1,577 & 1,762 & 2,729 & 5,005 & 2,450 & 1,105 & 1,401 \\
\hline Other liabilities & 359 & 307 & 901 & 692 & 602 & 457 & 432 \\
\hline
\end{tabular}

Source: Bank of Botswana.

1/ Effective January 1997, in accordance with the new Bank of Botswana Act, the balance sheet was reclassified, resulting in adjustments in the Pula fund and government deposits, with corresponding adjustments in the liquidity portfolio and revaluation reserves. 
Table 20. Botswana: Summary Accounts of Commercial Banks, 1998-2004

(In millions of pula; end of period)

\begin{tabular}{|c|c|c|c|c|c|c|c|}
\hline & 1998 & 1999 & 2000 & 2001 & 2002 & 2003 & 2004 \\
\hline Commercial bank reserves & 331 & 353 & 229 & 263 & 311 & 397 & 470 \\
\hline Cash & 145 & 203 & 180 & 220 & 289 & 285 & 274 \\
\hline Balances at Bank of Botswana & 186 & 150 & 50 & 43 & 22 & 111 & 196 \\
\hline Foreign assets & 1,417 & 1,346 & 1,435 & 2,231 & 1,555 & 1,772 & 1,485 \\
\hline Claims on monetary authorities & 1,322 & 1,718 & 1,197 & 1,874 & 1,732 & 2,229 & 2,749 \\
\hline Credit to domestic economy & 2,742 & 4,061 & 4,805 & 5,395 & 6,617 & 7,202 & 8,441 \\
\hline Claims on local governments & 14 & 15 & 2 & 1 & 0 & 0 & 0 \\
\hline Claims on parastatals & 267 & 528 & 458 & 480 & 462 & 381 & 433 \\
\hline $\begin{array}{l}\text { Claims on private sector } \\
\text { Of which }\end{array}$ & 2,461 & 3,518 & 4,344 & 4,915 & 6,155 & 6,821 & 8,007 \\
\hline Claims on households & 1,389 & 1,995 & 2,430 & 2,948 & 3,561 & 3,910 & 4,948 \\
\hline Other assets & 1,722 & 1,641 & 1,715 & 1,350 & 1,158 & 1,568 & 1,903 \\
\hline Fixed assets & 129 & 159 & 167 & 175 & 195 & 205 & 205 \\
\hline Others $1 /$ & 1,592 & 1,482 & 1,549 & 1,174 & 963 & 1,363 & 1,697 \\
\hline Assets $=$ liabilities & 7,533 & 9,118 & 9,381 & 11,113 & 11,374 & 13,168 & 15,048 \\
\hline Demand deposits of the public & 1,160 & 1,371 & 1,470 & 1,869 & 2,054 & 2,290 & 2,989 \\
\hline $\begin{array}{l}\text { Time and savings deposits } \\
\text { Savings deposits } \\
\text { Time deposits }\end{array}$ & 4,183 & 5,282 & 5,248 & 7,082 & 6,698 & 7,924 & 8,253 \\
\hline Liabilities to other banks & 172 & 161 & 225 & 368 & 292 & 421 & 333 \\
\hline Liabilities to Bank of Botswana & 0 & 0 & 0 & 0 & 64 & 1 & 4 \\
\hline Government deposits & 29 & 66 & 107 & 61 & 58 & 148 & 433 \\
\hline Capital and reserves & 568 & 732 & 843 & 1,042 & 1,102 & 1,346 & 1,395 \\
\hline Other liabilities & 1,421 & 1,506 & 1,489 & 691 & 1,107 & 1,038 & 1,640 \\
\hline
\end{tabular}

Source: Bank of Botswana.

1/ Comprises other investments: balances due from domestic banks, bills purchased and discounted, Bank of Botswana Certificates, and other unclassified assets. 
Table 21. Botswana: Selected Financial Ratios and Aggregates of Commercial Banks, 1998-2004 (In millions of pula, unless otherwise indicated; end of period)

\begin{tabular}{|c|c|c|c|c|c|c|c|}
\hline & 1998 & 1999 & 2000 & 2001 & 2002 & 2003 & 2004 \\
\hline \multicolumn{8}{|l|}{ Liquid assets $1 /$} \\
\hline Required & 449 & 528 & 581 & 676 & 812 & 899 & 1,006 \\
\hline Actual & 1,917 & 2,242 & 1,609 & 2,613 & 2,188 & 2,547 & 3,237 \\
\hline Excess & 1,468 & 1,714 & 1,028 & 1,937 & 1,376 & 1,649 & 2,231 \\
\hline Ratio of actual to required & 4.3 & 4.2 & 2.8 & 3.9 & 2.7 & 2.8 & 3.2 \\
\hline \multicolumn{8}{|l|}{ Primary reserves $2 /$} \\
\hline Required & 146 & 172 & 189 & 220 & 264 & 292 & 327 \\
\hline Actual & 332 & 322 & 238 & 263 & 286 & 403 & 523 \\
\hline Excess & 186 & 150 & 50 & 43 & 22 & 111 & 196 \\
\hline Ratio of actual to required & 2.3 & 1.9 & 1.3 & 1.2 & 1.1 & 1.4 & 1.6 \\
\hline Deposit liabilities & 5,424 & 6,756 & 6,912 & 9,233 & 8,983 & 10,574 & 11,876 \\
\hline Credit & 2,965 & 4,191 & 4,933 & 5,462 & 6,628 & 7,289 & 8,460 \\
\hline Ratio of credit to deposit liabilities & 0.5 & 0.6 & 0.7 & 0.6 & 0.7 & 0.7 & 0.7 \\
\hline
\end{tabular}

Source: Bank of Botswana.

1/ Required liquid assets are 10 percent of commercial banks' daily average deposit balances. Eligible liquid assets include cash, current account balance with the Bank of Botswana $(\mathrm{BoB})$ in excess of the primary required reserve, balances due from domestic banks, foreign notes and coins, $\mathrm{BoB}$ certificates, and private sector bills eligible for discount at the $\mathrm{BoB}$.

2/ Primary required reserves, consisting of current account balances with the $\mathrm{BoB}$, are 3.25 percent of average daily deposit balances. 
Table 22. Botswana: Selected Interest Rates, 1998-2004

(In percent; end of period)

\begin{tabular}{|c|c|c|c|c|c|c|c|}
\hline & 1998 & 1999 & 2000 & 2001 & 2002 & 2003 & 2004 \\
\hline \multicolumn{8}{|l|}{ Bank of Botswana } \\
\hline Lending rate (bank rate) & 12.5 & 13.3 & 14.3 & 14.3 & 15.3 & 14.3 & 14.3 \\
\hline \multicolumn{8}{|l|}{ Public Debt Service Fund 1/ } \\
\hline Financial parastatals & 12.1 & 12.1 & 13.8 & 13.8 & 13.8 & 13.8 & $\ldots$ \\
\hline Nonfinancial parastatals & 14.6 & 14.6 & 16.3 & 16.3 & 16.3 & 16.3 & $\ldots$ \\
\hline \multicolumn{8}{|l|}{ Commercial banks } \\
\hline \multicolumn{8}{|l|}{ Deposit rates } \\
\hline Savings accounts & 7.1 & 7.3 & 8.7 & 8.4 & 8.4 & 7.7 & 7.5 \\
\hline Call deposits & 9.1 & 8.7 & 9.7 & 9.5 & 10.0 & 9.6 & 8.8 \\
\hline 31 days' notice & 8.1 & 8.5 & 8.5 & 9.3 & 10.0 & 9.6 & 8.3 \\
\hline 88 days' notice & 8.5 & 9.2 & 10.2 & 9.8 & 10.2 & 9.5 & 9.1 \\
\hline \multicolumn{8}{|l|}{ Fixed deposits } \\
\hline 6 months & 9.0 & 9.8 & 10.3 & 10.4 & 10.8 & 10.5 & 9.6 \\
\hline 12 months & 9.1 & 10.4 & 10.7 & 10.6 & 11.0 & 10.3 & 10.1 \\
\hline Prime lending rate & 14.0 & 14.8 & 15.8 & 15.8 & 16.8 & 15.8 & 15.8 \\
\hline \multicolumn{8}{|l|}{ Botswana Building Society } \\
\hline \multicolumn{8}{|l|}{ Deposit rates } \\
\hline Indefinite period paid-up shares & 9.0 & 8.5 & 10.0 & 10.0 & 10.0 & 12.0 & 12.0 \\
\hline Subscription shares & 8.0 & 7.0 & 8.5 & 8.5 & 8.5 & 8.5 & 8.5 \\
\hline \multicolumn{8}{|l|}{ Fixed-time deposits } \\
\hline Ordinary savings accounts & 2.0 & 2.0 & 2.5 & 2.5 & 2.5 & 3.0 & 3.0 \\
\hline Special savings accounts & 7.0 & 7.0 & 7.5 & 7.5 & 7.5 & 10.0 & 10.0 \\
\hline \multicolumn{8}{|l|}{ Lending rates } \\
\hline Mortgage loans 2/ & 14.0 & 14.0 & 14.5 & 14.5 & 14.5 & 15.0 & 15.0 \\
\hline Short-term loans 3 / & 17.0 & 17.0 & 17.0 & 17.0 & 17.0 & 17.0 & 17.0 \\
\hline \multicolumn{8}{|l|}{ Botswana Savings Bank } \\
\hline Ordinary savings accounts & 3.5 & 3.5 & 3.5 & 3.5 & 3.5 & 4.0 & 4.0 \\
\hline Special savings accounts & 7.5 & 7.5 & 7.5 & 7.5 & 7.5 & 8.0 & 8.0 \\
\hline
\end{tabular}

Source: Bank of Botswana.

1/ In 1991, a two-tier rate structure was introduced, with the lower rate applying to financial parastatals and the higher rate to nonfinancial parastatals.

2/ Loans over P 50,000 are charged an additional percentage point.

$3 /$ Interest rates on short-term loans vary according to the security offered by the borrower. 
Table 23. Botswana: Distribution of Commercial Bank Credit by Economic Activity, 1998-2004

\begin{tabular}{|c|c|c|c|c|c|c|}
\hline & 1998 & 1999 & 2001 & 2002 & 2003 & 2004 \\
\hline & \multicolumn{6}{|c|}{ (In millions of pula; end of period) } \\
\hline Agriculture & 29 & 19 & 51 & 44 & 54 & 119 \\
\hline Mining & 59 & 182 & 39 & 128 & 116 & 40 \\
\hline Manufacturing & 192 & 220 & 264 & 330 & 389 & 352 \\
\hline Construction & 135 & 83 & 131 & 209 & 222 & 227 \\
\hline Electricity and water & 16 & 112 & 42 & 56 & 51 & 86 \\
\hline Transport and communications & 145 & 208 & 181 & 118 & 123 & 284 \\
\hline Trade & 261 & 212 & 390 & 579 & 849 & 428 \\
\hline Business services & 387 & 526 & 801 & 984 & 982 & 1,236 \\
\hline Finance & 2 & 12 & 37 & 25 & 26 & 22 \\
\hline Other business & 78 & 80 & 98 & 133 & 187 & 284.3 \\
\hline Financial parastatals & 267 & 528 & 480 & 462 & 381 & 433.3 \\
\hline Local government & 14 & 15 & 1 & 0 & 0 & 0 \\
\hline Central government & 0 & 0 & 0 & 0 & 0 & 0 \\
\hline Households & 1,380 & 1,995 & 2,948 & 3,561 & 3,910 & 4,948 \\
\hline \multirow[t]{2}{*}{ Total } & 2,965 & 4,192 & 5,462 & 6,628 & 7,289 & 8,460 \\
\hline & \multicolumn{6}{|c|}{ (In percent of total) } \\
\hline Agriculture & 1.0 & 0.5 & 0.9 & 0.7 & 0.7 & 1.4 \\
\hline Mining & 2.0 & 4.3 & 0.7 & 1.9 & 1.6 & 0.5 \\
\hline Manufacturing & 6.5 & 5.2 & 4.8 & 5.0 & 5.3 & 4.2 \\
\hline Construction & 4.6 & 2.0 & 2.4 & 3.2 & 3.0 & 2.7 \\
\hline Electricity and water & 0.5 & 2.7 & 0.8 & 0.8 & 0.7 & 1.0 \\
\hline Transport and communications & 4.9 & 5.0 & 3.3 & 1.8 & 1.7 & 3.4 \\
\hline Trade & 8.8 & 5.1 & 7.1 & 8.7 & 11.6 & 5.1 \\
\hline Business services & 13.1 & 12.5 & 14.7 & 14.9 & 13.5 & 14.6 \\
\hline Finance & 0.1 & 0.3 & 0.7 & 0.4 & 0.4 & 0.3 \\
\hline Other business & 2.6 & 1.9 & 1.8 & 2.0 & 2.6 & 3.4 \\
\hline Financial parastatals & 9.0 & 12.6 & 8.8 & 7.0 & 5.2 & 5.1 \\
\hline Local government & 0.5 & 0.4 & 0.0 & 0.0 & 0.0 & 0.0 \\
\hline Central government & 0.0 & 0.0 & 0.0 & 0.0 & 0.0 & 0.0 \\
\hline Households & 46.5 & 47.6 & 54.0 & 53.7 & 53.6 & 58.5 \\
\hline Total & 100.0 & 100.0 & 100.0 & 100.0 & 100.0 & 100.0 \\
\hline
\end{tabular}

Source: Bank of Botswana. 
Table 24. Botswana: Sources of Commercial Bank Deposits, 1998-2004

\begin{tabular}{|c|c|c|c|c|c|c|c|}
\hline & 1998 & 1999 & 2000 & 2001 & 2002 & 2003 & 2004 \\
\hline & \multicolumn{7}{|c|}{ (In millions of pula; end of period) } \\
\hline Government & 339 & 346 & 470 & 669 & 602 & 1,098 & 1,063 \\
\hline Central & 29 & 66 & 106 & 61 & 58 & 148 & 433 \\
\hline Local & 310 & 280 & 364 & 609 & 544 & 950 & 630 \\
\hline Parastatals & 685 & 855 & 617 & 823 & 780 & 969 & 1,166 \\
\hline Private enterprises & 3,371 & 3,926 & 3,425 & 5,571 & 5,109 & 5,249 & 7,147 \\
\hline Households & 1,030 & 1,629 & 2,401 & 2,171 & 2,492 & 3,259 & 2,500 \\
\hline \multirow[t]{2}{*}{ Total } & 5,424 & 6,757 & 6,912 & 9,233 & 8,983 & 10,574 & 11,876 \\
\hline & \multicolumn{7}{|c|}{ (In percent of total) } \\
\hline Government & 6.3 & 5.1 & 6.8 & 7.2 & 6.7 & 10.4 & 8.9 \\
\hline Central & 0.5 & 1.0 & 1.5 & 0.7 & 0.6 & 1.4 & 3.6 \\
\hline Local & 5.7 & 4.1 & 5.3 & 6.6 & 6.1 & 9.0 & 5.3 \\
\hline Parastatals & 12.6 & 12.7 & 8.9 & 8.9 & 8.7 & 9.2 & 9.8 \\
\hline Private enterprises & 62.1 & 58.1 & 49.5 & 60.3 & 56.9 & 49.6 & 60.2 \\
\hline Households & 19.0 & 24.1 & 34.7 & 23.5 & 27.7 & 30.8 & 21.1 \\
\hline Total & 100.0 & 100.0 & 100.0 & 100.0 & 100.0 & 100.0 & 100.0 \\
\hline
\end{tabular}

Source: Bank of Botswana. 
Table 25. Botswana: Auctions of Bank of Botswana Certificates, 2002-04 1/

\begin{tabular}{|c|c|c|c|c|}
\hline & $\begin{array}{r}\text { Allotted } \\
\text { (Millions of pula) }\end{array}$ & $\begin{array}{r}\text { Reserved for } \\
\text { Bank of } \\
\text { Botswana } \\
\text { (Millions of pula) }\end{array}$ & $\begin{array}{r}\text { Stop-Out } \\
\text { Price 2/ } \\
\text { (In pula) }\end{array}$ & $\begin{array}{r}\text { Effective } \\
\text { Interest Rate } \\
\text { (In percent) }\end{array}$ \\
\hline \multicolumn{5}{|l|}{2002} \\
\hline January & 1,577 & 371 & 97.0 & 12.8 \\
\hline February & 2,225 & 415 & 97.0 & 12.8 \\
\hline March & 1,256 & 554 & 97.0 & 12.8 \\
\hline April & 1,885 & 845 & 97.1 & 12.8 \\
\hline May & 2,485 & 289 & 97.0 & 12.8 \\
\hline June & 1,859 & 831 & 97.0 & 12.8 \\
\hline July & 2,070 & 1,250 & 97.0 & 12.8 \\
\hline August & 2,376 & 914 & 97.0 & 12.8 \\
\hline September & 2,698 & 2,842 & 97.0 & 12.8 \\
\hline October & 2,675 & 925 & 96.4 & 13.3 \\
\hline November & 2,620 & 1,075 & 96.8 & 14.1 \\
\hline December & 2,747 & 984 & 96.7 & 14.3 \\
\hline \multicolumn{5}{|l|}{2003} \\
\hline January & 2,868 & $\ldots$ & 96.7 & 14.3 \\
\hline February & 2,490 & 59 & 96.7 & 14.3 \\
\hline March & 2,059 & 801 & $96.720-96.725$ & $14.29-14.31$ \\
\hline April & 4,343 & 1,213 & $96.725-96.730$ & $14.27-14.29$ \\
\hline May & 1,921 & 62 & 96.7 & 14.3 \\
\hline June & 2,608 & 817 & $96.700-96.740$ & $14.22-14.27$ \\
\hline July & 3,952 & $\ldots$ & $96.725-96.740$ & $14.22-14.29$ \\
\hline August & 3,298 & $\ldots$ & $96.25-96.735$ & $14.24-14.29$ \\
\hline September & 3,265 & 533 & $96.760-96.790$ & $14.03-14.15$ \\
\hline October & 3,258 & 337 & $96.760-96.875$ & $13.58-1412$ \\
\hline November & 2,522 & 288 & $96.895-96.940$ & $13.28-13.49$ \\
\hline December & 3,194 & $\ldots$ & $96.950-96.990$ & $13.04-13.23$ \\
\hline \multicolumn{5}{|l|}{2004} \\
\hline January & 3,655 & $\ldots$ & $96.985-97.010$ & $12.95-13.06$ \\
\hline February & 2,508 & 326 & $97.010-97.040$ & $12.81-12.95$ \\
\hline March & 3,229 & $\ldots$ & $96.990-97.015$ & $12.92-13.04$ \\
\hline April & 3,216 & 623 & $97.000-97.025$ & $12.88-12.99$ \\
\hline May & 2,710 & 649 & $96.990-97.000$ & $12.99-13.04$ \\
\hline June & 3,152 & $\ldots$ & $96.990-97.000$ & $12.99-13.04$ \\
\hline July & 3,357 & $\ldots$ & 97.0 & 13.0 \\
\hline August & 3,679 & $\ldots$ & 97.0 & 13.0 \\
\hline September & 2,266 & $\ldots$ & 97.0 & 13.0 \\
\hline October & 3,500 & $\ldots$ & $97.000-97.005$ & 12.97-12.99 \\
\hline November & 3,697 & 695 & $99.565-97.010$ & $12.04-1295$ \\
\hline December & 4,033 & 1,067 & $99.565-97.015$ & $12.04-12.92$ \\
\hline
\end{tabular}

Source: Bank of Botswana.

1/ In any month with more than one auction, the stop-out price and interest rates are arithmetic averages.

2/ The stop-out price is the price below which no bid for Bank of Botswana Certificates will be entertained by the Bank of Botswana. 
Table 26. Botswana: Value of Outstanding Bank of Botswana Certificates, 2002-04 1/

(In millions of pula; end of period)

\begin{tabular}{|c|c|c|c|c|}
\hline & $\begin{array}{r}\text { Commercial } \\
\text { Banks }\end{array}$ & $\begin{array}{l}\text { Other Financial } \\
\text { Institutions }\end{array}$ & $\begin{array}{r}\text { Other Private } \\
\text { Sector }\end{array}$ & Total \\
\hline \multicolumn{5}{|l|}{2002} \\
\hline January & 3,445 & 689 & 904 & 5,038 \\
\hline February & 3,907 & 703 & 1,076 & 5,686 \\
\hline March & 3,204 & 830 & 610 & 4,644 \\
\hline April & 4,257 & 861 & 804 & 5,922 \\
\hline May & 4,146 & 942 & 667 & 5,755 \\
\hline June & 4,311 & 1,087 & 704 & 6,102 \\
\hline July & 4,399 & 1,086 & 590 & 6,075 \\
\hline August & 4,634 & 1,174 & 534 & 6,342 \\
\hline September & 5,329 & 1,266 & 431 & 7,026 \\
\hline October & 5,762 & 1,493 & 735 & 7,990 \\
\hline November & 5,612 & 1,698 & 674 & 7,984 \\
\hline December & 5,239 & 1,819 & 606 & 7,664 \\
\hline \multicolumn{5}{|l|}{2003} \\
\hline January & 5,632 & 1,932 & 428 & 7,992 \\
\hline February & 5,779 & 1,938 & 351 & 8,068 \\
\hline March & 5,461 & 2,024 & 240 & 7,726 \\
\hline April & 6,807 & 2,834 & 169 & 9,809 \\
\hline May & 5,991 & 1,881 & 1,010 & 8,882 \\
\hline June & 5,754 & 1,956 & 887 & 8,597 \\
\hline July & 6,032 & 2,220 & 864 & 9,117 \\
\hline August & 6,667 & 2,249 & 1,099 & 10,015 \\
\hline September & 6,607 & 2,087 & 1,155 & 9,850 \\
\hline October & 7,316 & 2,144 & 1,061 & 10,521 \\
\hline November & 6,583 & 2,049 & 887 & 9,519 \\
\hline December & 5,959 & 1,905 & 875 & 8,739 \\
\hline \multicolumn{5}{|l|}{2004} \\
\hline January & 6,537 & 1,822 & 889 & 9,248 \\
\hline February & 5,800 & 1,996 & 983 & 8,778 \\
\hline March & 5,918 & 1,763 & 892 & 8,573 \\
\hline April & 5,992 & 1,659 & 1,461 & 9,112 \\
\hline May & 6,995 & 1,511 & 1,412 & 9,918 \\
\hline June & 6,005 & 1,422 & 1,757 & 9,184 \\
\hline July & 6,228 & 1,225 & 1,788 & 9,241 \\
\hline August & 6,543 & 1,116 & 1,795 & 9,455 \\
\hline September & 6,543 & 875 & 1,790 & 9,208 \\
\hline October & 6,541 & 972 & 1,852 & 9,365 \\
\hline November & 6,453 & 975 & 2,093 & 9,522 \\
\hline December & 6,626 & 1,008 & 2,015 & 9,649 \\
\hline
\end{tabular}

Sources: Bank of Botswana.

1/ Total market value, excluding interest. 
Table 27. Botswana: Financial System Structure, 2004

\begin{tabular}{|c|c|c|c|}
\hline & $\begin{array}{r}\text { Assets } \\
\text { (In billions of pula) }\end{array}$ & $\begin{array}{l}\text { Percent of } \\
\text { total assets }\end{array}$ & $\begin{array}{r}\text { Percent of } \\
\text { GDP }\end{array}$ \\
\hline Total onshore financial system & 39.0 & 100.0 & 92.6 \\
\hline Banks - onshore & 15.6 & 40.0 & 37.0 \\
\hline Domestic commercial banks & $\ldots$ & $\ldots$ & $\ldots$ \\
\hline Foreign commercial banks & 14.8 & 38.1 & 35.3 \\
\hline State-owned commercial banks & $\ldots$ & $\ldots$ & $\ldots$ \\
\hline Merchant banks & 0.7 & 1.9 & 1.8 \\
\hline Institutional Investors & 10.0 & 25.7 & 23.8 \\
\hline Insurance companies $1 /$ & 0.6 & 1.6 & 1.5 \\
\hline Pension funds $2 /$ & 6.8 & 17.4 & 16.1 \\
\hline Collective investment undertaking (CIU) & 2.6 & 6.8 & 6.3 \\
\hline Other nonbank & 13.4 & 34.3 & 31.7 \\
\hline Botswana Development Corporation & 1.2 & 3.0 & 2.8 \\
\hline National Development Bank & 0.5 & 1.4 & 1.3 \\
\hline Botswana Savings Bank & 0.2 & 0.6 & 0.5 \\
\hline Botswana Building Society & 0.6 & 1.5 & 1.4 \\
\hline Leasing companies & $\ldots$ & $\ldots$ & $\ldots$ \\
\hline Securities firms & 10.9 & 27.9 & 25.8 \\
\hline Total offshore financial system & 3.4 & 100.0 & 8.1 \\
\hline Banks - offshore & 0.0 & 0.7 & 0.1 \\
\hline Insurance companies - offshore & $\ldots$ & $\ldots$ & $\ldots$ \\
\hline Other offshore NBFIs & 3.4 & 99.2 & 8.1 \\
\hline Total financial system & 42.4 & & 100.7 \\
\hline
\end{tabular}

Source: Botswana authorities.

1/ Data refers to end-2003.

2 / Refers to funds invested domestically which are 43 percent of total pension funds. 
Table 28. Botswana: Balance of Payments, 1998-2004 1/

(In millions of U.S. dollars, unless otherwise indicated)

\begin{tabular}{|c|c|c|c|c|c|c|c|}
\hline & 1998 & 1999 & 2000 & 2001 & 2002 & 2003 & $\begin{array}{c}2004 \\
\text { Est. }\end{array}$ \\
\hline Current account balance & 205 & 619 & 547 & 600 & 117 & 502 & 893 \\
\hline Trade balance & 78 & 786 & 904 & 714 & 618 & 958 & 633 \\
\hline $\begin{array}{l}\text { Exports, f.o.b. } \\
\text { Of which }\end{array}$ & 2,073 & 2,661 & 2,682 & 2,325 & 2,353 & 2,976 & 3,502 \\
\hline Diamonds & 1,480 & 2,122 & 2,236 & 1,936 & 1,978 & 2,374 & 2,703 \\
\hline Imports, f.o.b & $-1,995$ & $-1,876$ & $-1,778$ & $-1,611$ & $-1,735$ & $-2,018$ & $-2,870$ \\
\hline \multicolumn{8}{|l|}{ Of which } \\
\hline Food, beverages, and tobacco & -297 & -306 & -294 & -254 & -198 & -273 & -319 \\
\hline Chemical and rubber products & -201 & -204 & -203 & -187 & -129 & -202 & -236 \\
\hline Metal and metal products & -228 & -190 & -151 & -140 & -211 & -151 & -176 \\
\hline Machinery and electrical equipment & -480 & -464 & -463 & -357 & -79 & -385 & -449 \\
\hline Services & -235 & -156 & -223 & -174 & -29 & -5 & -18 \\
\hline Transportation & -165 & -162 & -174 & -155 & -167 & -176 & -267 \\
\hline Travel & 72 & 87 & 25 & 26 & 136 & 228 & 263 \\
\hline Other services & -143 & -81 & -73 & -45 & 3 & -57 & -15 \\
\hline Income & 120 & -263 & -352 & -138 & -700 & -718 & -364 \\
\hline Compensation of employees & -15 & -26 & -37 & -37 & -39 & -55 & -70 \\
\hline Investment income & 135 & -236 & -315 & -101 & -662 & -663 & -294 \\
\hline \multicolumn{8}{|l|}{ Of which } \\
\hline Earnings on reserves & 531 & 348 & 282 & 285 & 265 & 246 & 240 \\
\hline Current transfers & 242 & 252 & 218 & 198 & 228 & 268 & 489 \\
\hline Capital and financial account & -172 & -223 & -164 & -506 & -202 & -358 & -725 \\
\hline Capital account & 32 & 21 & 38 & 6 & 16 & 23 & 5 \\
\hline Financial account & -204 & -244 & -202 & -512 & -218 & -380 & -730 \\
\hline Direct investment & 92 & 35 & 55 & -351 & 362 & 213 & -239 \\
\hline Portfolio investment & -52 & -35 & -43 & -63 & -414 & -522 & -398 \\
\hline Other investment & -244 & -244 & -214 & -99 & -165 & -70 & -93 \\
\hline \multicolumn{8}{|l|}{ Of which } \\
\hline Net government long-term borrowing & 22 & -26 & -32 & -17 & -23 & -24 & -29 \\
\hline Other net private long-term borrowing & 28 & 2 & 2 & 2 & 2 & 3 & 4 \\
\hline Short-term borrowing & 20 & 46 & 46 & 76 & 71 & 93 & 63 \\
\hline Net errors and omissions $2 /$ & 233 & -107 & -293 & -516 & -339 & -280 & 161 \\
\hline Reserve assets (increase -) 2/ & -266 & -288 & -89 & 421 & 424 & 135 & -328 \\
\hline \multicolumn{8}{|l|}{ Memorandum items: } \\
\hline Current account balance (in percent of GDP) & 4.1 & 12.3 & 10.4 & 11.5 & 2.2 & 6.5 & 9.8 \\
\hline Trade balance (in percent of GDP) & 1.6 & 15.6 & 17.2 & 13.7 & 11.4 & 12.3 & 7.0 \\
\hline End-of-year gross official reserves & 5,941 & 6,229 & 6,319 & 5,897 & 5,474 & 5,339 & 5,667 \\
\hline (in months of imports of goods and services) & 28.2 & 31.1 & 32.6 & 33.0 & 29.1 & 24.0 & 18.5 \\
\hline \multicolumn{8}{|l|}{ Exchange rates } \\
\hline U.S. dollars per pula (period average) & 0.24 & 0.22 & 0.20 & 0.17 & 0.16 & 0.20 & 0.22 \\
\hline U.S. dollars per pula (end of period) & 0.22 & 0.22 & 0.19 & 0.14 & 0.18 & 0.23 & 0.23 \\
\hline
\end{tabular}

Sources: Botswana authorities; and IMF staff estimates.

1/ Based on pula-denominated estimates converted at period-average exchange rate.

2/ Includes valuation adjustment. 
Table 29. Botswana: Value of Principal Exports and Imports, 1998-2004

\begin{tabular}{|c|c|c|c|c|c|c|c|}
\hline & 1998 & 1999 & 2000 & 2001 & 2002 & 2003 & $\begin{array}{r}2004 \\
\text { Est. }\end{array}$ \\
\hline & \multicolumn{7}{|c|}{ (In millions of U.S. dollars) } \\
\hline Diamonds & 1,480 & 2,122 & 2,236 & 1,936 & 1,978 & 2,374 & 2,703 \\
\hline Nondiamond & 593 & 539 & 446 & 388 & 375 & 602 & 799 \\
\hline Meat & 51 & 55 & 50 & 66 & 41 & 50 & 59 \\
\hline Copper nickel & 73 & 88 & 108 & 70 & 76 & 141 & 166 \\
\hline Textiles & 72 & 54 & 48 & 33 & 29 & 60 & 62 \\
\hline Soda ash & 40 & 43 & 41 & 36 & 42 & 47 & 54 \\
\hline Other & 358 & 300 & 200 & 183 & 186 & 305 & 459 \\
\hline Total exports, f.o.b. & 2,073 & 2,661 & 2,682 & 2,325 & 2,353 & 2,976 & 3,502 \\
\hline Food, beverages, and tobacco & 297 & 306 & 294 & 254 & 198 & 273 & 319 \\
\hline Wood and paper products & 155 & 177 & 161 & 160 & 342 & 172 & 201 \\
\hline Textile and footwear & 136 & 129 & 121 & 85 & 124 & 91 & 107 \\
\hline Chemical and rubber products & 201 & 204 & 203 & 188 & 129 & 202 & 236 \\
\hline Fuel & 103 & 107 & 103 & 122 & 295 & 362 & 495 \\
\hline Metal and metal products & 228 & 190 & 151 & 140 & 211 & 151 & 176 \\
\hline Machinery and electrical equipment & 480 & 464 & 463 & 357 & 79 & 385 & 449 \\
\hline Vehicle and transport equipment & 368 & 297 & 258 & 221 & 261 & 238 & 278 \\
\hline Other goods & 296 & 327 & 332 & 289 & 125 & 311 & 363 \\
\hline \multirow[t]{2}{*}{ Total imports, c.i.f. } & 2,265 & 2,200 & 2,085 & 1,816 & 1,765 & 2,185 & 2,624 \\
\hline & \multicolumn{7}{|c|}{ (In percent of total) } \\
\hline Diamonds & 71.4 & 79.7 & 83.4 & 83.3 & 84.1 & 79.8 & 77.2 \\
\hline Nondiamond & 28.6 & 20.3 & 16.6 & 16.7 & 15.9 & 20.2 & 22.8 \\
\hline Meat & 2.4 & 2.1 & 1.9 & 2.9 & 1.8 & 1.7 & 1.7 \\
\hline Copper nickel & 3.5 & 3.3 & 4.0 & 3.0 & 3.3 & 4.7 & 4.7 \\
\hline Textiles & 3.5 & 2.0 & 1.8 & 1.4 & 1.2 & 2.0 & 1.8 \\
\hline Soda ash & 1.9 & 1.6 & 1.5 & 1.5 & 1.8 & 1.6 & 1.5 \\
\hline Other & 17.3 & 11.3 & 7.4 & 7.9 & 7.9 & 10.2 & 13.1 \\
\hline Total exports, f.o.b. & 100.0 & 100.0 & 100.0 & 100.0 & 100.0 & 100.0 & 100.0 \\
\hline Food, beverages, and tobacco & 13.1 & 13.9 & 14.1 & 14.0 & 11.2 & 12.5 & 12.2 \\
\hline Wood and paper products & 6.9 & 8.1 & 7.7 & 8.8 & 19.4 & 7.9 & 7.6 \\
\hline Textile and footwear & 6.0 & 5.9 & 5.8 & 4.7 & 7.0 & 4.2 & 4.1 \\
\hline Chemical and rubber products & 8.9 & 9.3 & 9.7 & 10.3 & 7.3 & 9.2 & 9.0 \\
\hline Fuel & 4.6 & 4.9 & 4.9 & 6.7 & 16.7 & 16.6 & 18.9 \\
\hline Metal and metal products & 10.1 & 8.6 & 7.2 & 7.7 & 12.0 & 6.9 & 6.7 \\
\hline Machinery and electrical equipment & 21.2 & 21.1 & 22.2 & 19.7 & 4.5 & 17.6 & 17.1 \\
\hline Vehicle and transport equipment & 16.3 & 13.5 & 12.4 & 12.2 & 14.8 & 10.9 & 10.6 \\
\hline Other goods & 13.1 & 14.8 & 15.9 & 15.9 & 7.1 & 14.2 & 13.8 \\
\hline Total imports, c.i.f. & 100.0 & 100.0 & 100.0 & 100.0 & 100.0 & 100.0 & 100.0 \\
\hline
\end{tabular}

Source: Botswana authorities; and IMF staff estimates. 
Table 30. Botswana: External Trade Indices, 1998/99-2002/03 1/

\begin{tabular}{|c|c|c|c|c|c|}
\hline & 1998/99 & $1999 / 00$ & $2000 / 01$ & $2001 / 02$ & $2002 / 03$ \\
\hline \multicolumn{6}{|l|}{ Values (in millions of pula) } \\
\hline Exports of goods & 8,560 & 13,637 & 15,714 & 12,993 & 12,847 \\
\hline Exports of services & 1,492 & 1,682 & 1,841 & 2,571 & 3,286 \\
\hline Total exports (goods and services) & 10,052 & 15,318 & 17,555 & 15,564 & 16,132 \\
\hline Imports of goods & $-8,571$ & $-8,866$ & $-8,965$ & $-9,732$ & $-8,273$ \\
\hline Imports of services & $-1,389$ & $-1,557$ & $-1,841$ & $-2,006$ & $-4,418$ \\
\hline Total imports (goods and services) & $-9,961$ & $-10,422$ & $-10,806$ & $-11,738$ & $-12,690$ \\
\hline \multicolumn{6}{|c|}{ Volume (1993/94 prices, millions of pula) } \\
\hline Exports of goods & 4,959 & 6,662 & 7,342 & 7,342 & 7,342 \\
\hline Exports of services & 994 & 1,032 & 1,143 & 1,143 & 1,143 \\
\hline Total exports (goods and services) & 5,952 & 7,694 & 8,485 & 8,485 & 8,485 \\
\hline Imports of goods & $-5,656$ & $-5,322$ & $-5,776$ & $-5,776$ & $-5,776$ \\
\hline Imports of services & -981 & $-1,391$ & $-1,509$ & $-1,509$ & $-1,509$ \\
\hline Total imports (goods and services) & $-6,637$ & $-6,713$ & $-7,285$ & $-7,285$ & $-7,285$ \\
\hline \multicolumn{6}{|l|}{ Price indices $(1993 / 94=100)$} \\
\hline Exports of goods & 172.6 & 204.7 & 214.0 & 177.0 & 175.0 \\
\hline Exports of services & 150.1 & 163.0 & 161.1 & 224.9 & 287.5 \\
\hline Total exports (goods and services) & 168.9 & 199.1 & 206.9 & 183.4 & 190.1 \\
\hline Imports of goods & 151.6 & 166.6 & 155.2 & 168.5 & 143.2 \\
\hline Imports of services & 141.6 & 111.9 & 122.0 & 133.0 & 292.8 \\
\hline Total imports (goods and services) & 150.1 & 155.3 & 148.3 & 161.1 & 174.2 \\
\hline
\end{tabular}

Source: National accounts data.

1/ Year beginning July 1. 
Table 31. Botswana: Direction of Trade, 1997-2004

\begin{tabular}{|c|c|c|c|c|c|c|c|c|}
\hline & 1997 & 1998 & 1999 & 2000 & 2001 & 2002 & 2003 & 2004 \\
\hline & & & & \multicolumn{5}{|c|}{ Est. } \\
\hline & \multicolumn{8}{|c|}{ (In millions of pula) } \\
\hline Exports, f.o.b. & 10,391 & 8,693 & 12,228 & 13,649 & 13,519 & 14,843 & 14,678 & 16,268 \\
\hline Southern African Customs Union (SACU) & 1,485 & 1,494 & 1,271 & 1,419 & 1,405 & 1,543 & 1,526 & 1,691 \\
\hline Zimbabwe & 383 & 249 & 291 & 325 & 322 & 353 & 349 & 387 \\
\hline Other Africa & 114 & 111 & 137 & 153 & 152 & 167 & 165 & 183 \\
\hline United Kingdom & 5,840 & 4,830 & 8,130 & 9,076 & 8,989 & 9,869 & 9,759 & 10,817 \\
\hline Other Europe & 2,444 & 1,870 & 2,221 & 2,479 & 2,455 & 2,695 & 2,666 & 2,954 \\
\hline United States & 102 & 90 & 86 & 97 & 96 & 105 & 104 & 115 \\
\hline All other & 23 & 48 & 91 & 102 & 101 & 111 & 110 & 121 \\
\hline Imports, c.i.f. & 8,256 & 9,804 & 10,164 & 9,047 & 9,370 & 10,944 & 9,951 & 13,330 \\
\hline SACU & 5,982 & 7,402 & 7,783 & 6,928 & 7,175 & 8,380 & 7,620 & 10,208 \\
\hline Zimbabwe & 368 & 375 & 397 & 353 & 366 & 427 & 388 & 520 \\
\hline Other Africa & 38 & 54 & 27 & 24 & 25 & 29 & 26 & 35 \\
\hline United Kingdom & 163 & 321 & 272 & 243 & 251 & 293 & 267 & 357 \\
\hline Other Europe & 580 & 644 & 664 & 591 & 612 & 715 & 650 & 871 \\
\hline Korea, Republic of & 785 & 457 & 264 & 235 & 243 & 284 & 258 & 346 \\
\hline United States & 89 & 135 & 188 & 167 & 173 & 202 & 184 & 247 \\
\hline \multirow[t]{2}{*}{ All other } & 251 & 418 & 569 & 506 & 524 & 612 & 557 & 746 \\
\hline & \multicolumn{8}{|c|}{ (In percent of total) } \\
\hline Exports, f.o.b. & 100.0 & 100.0 & 100.0 & 100.0 & 100.0 & 100.0 & 100.0 & 100.0 \\
\hline SACU & 14.3 & 17.2 & 10.4 & 10.4 & 10.4 & 10.4 & 10.4 & 10.4 \\
\hline Zimbabwe & 3.7 & 2.9 & 2.4 & 2.4 & 2.4 & 2.4 & 2.4 & 2.4 \\
\hline Other Africa & 1.1 & 1.3 & 1.1 & 1.1 & 1.1 & 1.1 & 1.1 & 1.1 \\
\hline United Kingdom & 56.2 & 55.6 & 66.5 & 66.5 & 66.5 & 66.5 & 66.5 & 66.5 \\
\hline Other Europe & 23.5 & 21.5 & 18.2 & 18.2 & 18.2 & 18.2 & 18.2 & 18.2 \\
\hline United States & 1.0 & 1.0 & 0.7 & 0.7 & 0.7 & 0.7 & 0.7 & 0.7 \\
\hline All other & 0.2 & 0.6 & 0.7 & 0.7 & 0.7 & 0.7 & 0.7 & 0.7 \\
\hline Imports, c.i.f. & 100.0 & 100.0 & 100.0 & 100.0 & 100.0 & 100.0 & 100.0 & 100.0 \\
\hline SACU & 72.5 & 75.5 & 76.6 & 76.6 & 76.6 & 76.6 & 76.6 & 76.6 \\
\hline Zimbabwe & 4.5 & 3.8 & 3.9 & 3.9 & 3.9 & 3.9 & 3.9 & 3.9 \\
\hline Other Africa & 0.5 & 0.5 & 0.3 & 0.3 & 0.3 & 0.3 & 0.3 & 0.3 \\
\hline United Kingdom & 2.0 & 3.3 & 2.7 & 2.7 & 2.7 & 2.7 & 2.7 & 2.7 \\
\hline Other Europe & 7.0 & 6.6 & 6.5 & 6.5 & 6.5 & 6.5 & 6.5 & 6.5 \\
\hline Korea, Republic of & 9.5 & 4.7 & 2.6 & 2.6 & 2.6 & 2.6 & 2.6 & 2.6 \\
\hline United States & 1.1 & 1.4 & 1.8 & 1.8 & 1.8 & 1.8 & 1.8 & 1.8 \\
\hline All other & 3.0 & 4.3 & 5.6 & 5.6 & 5.6 & 5.6 & 5.6 & 5.6 \\
\hline
\end{tabular}

Sources: Customs and Excise Department; and IMF staff estimates. 
Table 32. Botswana: Public Sector External Debt, 1998-2004

\begin{tabular}{|c|c|c|c|c|c|c|c|}
\hline & 1998 & 1999 & 2000 & 2001 & 2002 & 2003 & $\begin{array}{c}2004 \\
\text { Est. }\end{array}$ \\
\hline & \multicolumn{7}{|c|}{ (In millions of U.S. dollars; end of period) } \\
\hline Total external public debt $1 /$ & 441.5 & 523.1 & 452.3 & 347.4 & 533.6 & 494.0 & 418.9 \\
\hline Bilateral loans & 103.4 & 191.7 & 146.1 & 112.2 & 166.0 & 138.1 & 122.6 \\
\hline United States & 21.2 & 23.7 & 20.2 & 15.5 & 26.2 & 19.4 & 15.2 \\
\hline United Kingdom & 0.0 & 0.0 & 0.0 & 0.0 & 0.0 & 0.0 & 0.0 \\
\hline China & 18.3 & 23.5 & 27.1 & 20.8 & 34.7 & 31.2 & 27.8 \\
\hline France & 0.0 & 0.0 & 0.0 & 0.0 & 0.0 & 0.0 & 0.0 \\
\hline Belgium & 1.2 & 1.3 & 1.0 & 0.7 & 1.0 & 1.1 & 0.9 \\
\hline Kuwait & 13.2 & 13.5 & 9.8 & 7.5 & 15.7 & 13.0 & 10.3 \\
\hline Germany & 0.0 & 0.0 & 0.0 & 0.0 & 0.0 & 0.0 & 0.0 \\
\hline Nigeria & 0.0 & 0.0 & 0.0 & 0.0 & 0.0 & 0.0 & 0.0 \\
\hline Japan & 43.7 & 116.6 & 86.8 & 66.7 & 87.9 & 73.3 & 68.3 \\
\hline Saudi Arabia & 5.9 & 13.1 & 1.1 & 0.9 & 0.5 & 0.0 & 0.0 \\
\hline Multilateral loans & 331.6 & 325.2 & 287.6 & 220.9 & 347.6 & 343.5 & 296.4 \\
\hline African Development Bank 2/ & 188.5 & 183.5 & 78.9 & 64.4 & 84.1 & 190.7 & 110.8 \\
\hline African Development Fund & 0.0 & 0.0 & 83.3 & 60.1 & 91.4 & 68.4 & 57.0 \\
\hline Arab Bank for Economic Development in Africa & 18.7 & 20.2 & 17.8 & 13.7 & 28.3 & 23.2 & 17.4 \\
\hline European Economic Community & 0.0 & 0.0 & 0.0 & 0.0 & 0.0 & 0.0 & 0.0 \\
\hline European Investment Bank & 66.9 & 72.8 & 63.7 & 48.9 & 84.8 & 87.1 & 78.0 \\
\hline International Bank for Reconstruction and Development & 29.3 & 26.0 & 17.5 & 13.5 & 15.6 & 6.7 & 3.9 \\
\hline International Development Association & 9.0 & 10.1 & 8.6 & 6.6 & 14.2 & 9.4 & 7.6 \\
\hline International Fund for Agriculture Development & 0.0 & 0.0 & 0.0 & 0.0 & 0.0 & 0.0 & 0.0 \\
\hline Nordic Development Fund & 0.0 & 0.0 & 0.0 & 0.0 & 0.0 & 0.0 & 0.0 \\
\hline Nordic Investment Bank & 13.4 & 6.9 & 13.3 & 10.2 & 24.5 & 23.5 & 19.6 \\
\hline Organization of Petroleum Exporting Countries & 5.7 & 5.7 & 4.4 & 3.4 & 4.7 & 3.0 & 2.0 \\
\hline UN Capital Development Fund & 0.0 & 0.0 & 0.0 & 0.0 & 0.0 & 0.0 & 0.0 \\
\hline Commercial banks & 0.0 & 0.0 & 0.0 & 0.0 & 0.0 & 0.0 & $\ldots$ \\
\hline Export credits & 6.4 & 6.2 & 18.6 & 14.3 & 20.0 & 12.5 & $\ldots$ \\
\hline \multicolumn{8}{|l|}{ Memorandum items: } \\
\hline & \multicolumn{7}{|c|}{ (In percent) } \\
\hline Total external public debt & & & & & & & \\
\hline (in percent of GDP) & 9.4 & 10.4 & 9.1 & 8.0 & 8.5 & 5.7 & 4.5 \\
\hline (in percent of exports of goods and services) & 19.7 & 17.3 & 15.8 & 15.6 & 16.3 & 12.3 & 9.5 \\
\hline
\end{tabular}

Sources: Ministry of Finance and Development Planning; and IMF staff estimates.

1/ Disbursed government and government-guaranteed outstanding debt, with original maturity of one year and more.

2/ African Development Bank/Fund for 1999. 
Table 33. Botswana: External Debt-Service Payments and Disbursements, 1998-2004

\begin{tabular}{|c|c|c|c|c|c|c|c|}
\hline & 1998 & 1999 & 2000 & 2001 & 2002 & 2003 & $\begin{array}{r}2004 \\
\text { Est. }\end{array}$ \\
\hline & \multicolumn{7}{|c|}{ (In millions of U.S. dollars) } \\
\hline \multicolumn{8}{|l|}{ Central government } \\
\hline Disbursed outstanding debt & 556.3 & 530.5 & 498.1 & 451.2 & 431.6 & 425.3 & 427.9 \\
\hline Disbursements & 55.8 & 42.0 & 22.2 & 7.3 & 12.9 & 5.1 & 13.2 \\
\hline Principal repayments & 35.3 & 36.0 & 33.3 & 41.5 & 48.5 & 49.7 & 37.5 \\
\hline Interest payments & 32.0 & 33.4 & 31.8 & 17.9 & 20.2 & 17.4 & 41.5 \\
\hline Other charges & 0.2 & 0.1 & 0.0 & 0.0 & 0.0 & 0.0 & 0.0 \\
\hline Total debt service & 67.5 & 69.4 & 65.1 & 59.4 & 68.7 & 67.1 & 78.9 \\
\hline \multicolumn{8}{|l|}{ Public corporations } \\
\hline Disbursed outstanding debt & 69.7 & 32.1 & 23.9 & 19.6 & 15.3 & 17.2 & 13.5 \\
\hline Disbursements & 23.8 & 1.1 & 1.0 & 0.8 & 0.0 & 0.0 & 0.0 \\
\hline Principal repayments & 0.0 & 12.3 & 5.0 & 4.9 & 5.5 & 4.8 & 4.3 \\
\hline Interest payments & 4.6 & 6.3 & 2.9 & 2.4 & 2.8 & 2.4 & 1.9 \\
\hline Other charges & 0.0 & 0.0 & 0.0 & 0.0 & 0.0 & 0.0 & 0.0 \\
\hline Total debt service & 4.6 & 18.6 & 7.8 & 7.3 & 8.3 & 7.2 & 6.2 \\
\hline \multicolumn{8}{|l|}{ Public and publicly guaranteed } \\
\hline Disbursed outstanding debt & 626.0 & 562.6 & 522.1 & 570.8 & 447.0 & 442.6 & 441.3 \\
\hline Disbursements & 79.6 & 43.1 & 23.1 & 8.1 & 12.9 & 5.1 & 13.2 \\
\hline Principal repayments & 35.3 & 48.3 & 38.2 & 46.4 & 54.0 & 54.5 & 41.8 \\
\hline Interest payments & 36.6 & 39.7 & 34.7 & 20.2 & 23.0 & 19.8 & 43.4 \\
\hline Other charges & 0.2 & 0.1 & 0.0 & 0.0 & 0.0 & 0.0 & 0.0 \\
\hline Total debt service & 72.1 & 88.1 & 73.0 & 66.6 & 77.0 & 74.3 & 85.1 \\
\hline \multicolumn{8}{|l|}{ Memorandum items: } \\
\hline & \multicolumn{7}{|c|}{ (In percent of exports of goods and services) } \\
\hline Debt-service ratios & 3.0 & 2.9 & 2.4 & 2.5 & 2.7 & 2.1 & 2.0 \\
\hline Central government & 2.8 & 2.3 & 2.2 & 2.2 & 2.4 & 1.9 & 1.8 \\
\hline Public corporations & 0.2 & 0.6 & 0.3 & 0.3 & 0.3 & 0.2 & 0.1 \\
\hline
\end{tabular}

Sources: Ministry of Finance and Development Planning; and IMF staff estimates. 
Table 34. Botswana: Average Terms of New Public Sector External Borrowing, 1997-2004

\begin{tabular}{|c|c|c|c|c|c|c|c|c|}
\hline & 1997 & 1998 & 1999 & 2000 & 2001 & 2002 & 2003 & 2004 \\
\hline Interest rate (in percent a year) & 2.3 & 2.3 & 2.3 & 2.3 & $\ldots$ & $\ldots$ & $\ldots$ & ... \\
\hline Maturity (in years) & 14.5 & 14.5 & 14.5 & 14.5 & $\cdots$ & $\cdots$ & $\ldots$ & ... \\
\hline Grace period (in years) & 4.7 & 4.7 & 4.7 & 4.7 & $\ldots$ & $\ldots$ & $\ldots$ & $\ldots$ \\
\hline Grant element (in percent) & 40.7 & 40.7 & 40.7 & 40.7 & $\ldots$ & $\ldots$ & $\ldots$ & $\ldots$ \\
\hline
\end{tabular}

Source: Ministry of Finance and Development Planning. 
Table 35. Botswana: Developments in the Exchange Rate, 1990-2004 (Index, 1990=100, unless otherwise indicated; period average)

\begin{tabular}{|c|c|c|c|c|c|}
\hline & \multicolumn{2}{|c|}{ Effective Exchange Rate } & \multicolumn{3}{|c|}{ Bilateral Exchange Rates 1/ } \\
\hline & Real & Nominal & $\begin{array}{r}\text { South African } \\
\text { rand }\end{array}$ & U.S. dollar & $\begin{array}{r}\text { Zimbabwe } \\
\text { dollar }\end{array}$ \\
\hline 1990 & 100.00 & 100.00 & 1.39 & 0.54 & 1.32 \\
\hline 1991 & 96.03 & 96.80 & 1.37 & 0.49 & 1.79 \\
\hline 1992 & 97.20 & 93.80 & 1.35 & 0.47 & 2.42 \\
\hline 1993 & 100.81 & 91.90 & 1.35 & 0.41 & 2.68 \\
\hline 1994 & 99.85 & 88.40 & 1.32 & 0.37 & 3.04 \\
\hline 1995 & 100.94 & 86.80 & 1.31 & 0.36 & 3.13 \\
\hline 1996 & 99.40 & 82.50 & 1.29 & 0.30 & 3.01 \\
\hline 1997 & 96.99 & 79.48 & 1.26 & 0.27 & 3.32 \\
\hline 1998 & 97.86 & 79.27 & 1.31 & 0.24 & 5.60 \\
\hline 1999 & 99.43 & 78.09 & 1.32 & 0.22 & 8.28 \\
\hline 2000 & 103.24 & 78.21 & 1.36 & 0.20 & 8.71 \\
\hline 2001 & 108.14 & 80.64 & 1.47 & 0.17 & 9.42 \\
\hline $2002 \mathrm{I}$ & 116.31 & 86.63 & 1.69 & 0.15 & 8.09 \\
\hline $2002 \mathrm{II}$ & 109.88 & 76.94 & 1.64 & 0.16 & 8.90 \\
\hline 2002 III & 113.10 & 76.99 & 1.68 & 0.16 & 8.84 \\
\hline $2002 \mathrm{IV}$ & 113.05 & 77.11 & 1.59 & 0.18 & 9.55 \\
\hline $2003 \mathrm{I}$ & 113.84 & 77.26 & 1.56 & 0.17 & 107.4 \\
\hline 2003 II & 116.63 & 77.44 & 1.52 & 0.20 & 165.3 \\
\hline 2003 III & 118.39 & 77.49 & 1.51 & 0.21 & 167.7 \\
\hline $2003 \mathrm{IV}$ & 119.89 & 77.52 & 1.48 & 0.22 & 182.1 \\
\hline 2004 I & 114.51 & 73.64 & 1.42 & 0.21 & 332.1 \\
\hline 2004 II & 114.02 & 71.71 & 1.37 & 0.21 & 407.0 \\
\hline 2004 III & 115.11 & 71.71 & 1.36 & 0.21 & 410.7 \\
\hline 2004 IV & 115.80 & 71.71 & 1.33 & 0.23 & 444.6 \\
\hline
\end{tabular}

Source: IMF, International Financial Statistics .

1/ Foreign currency per Botswana pula. Increase in value indicates an appreciation of the Pula, 1990-2004. 


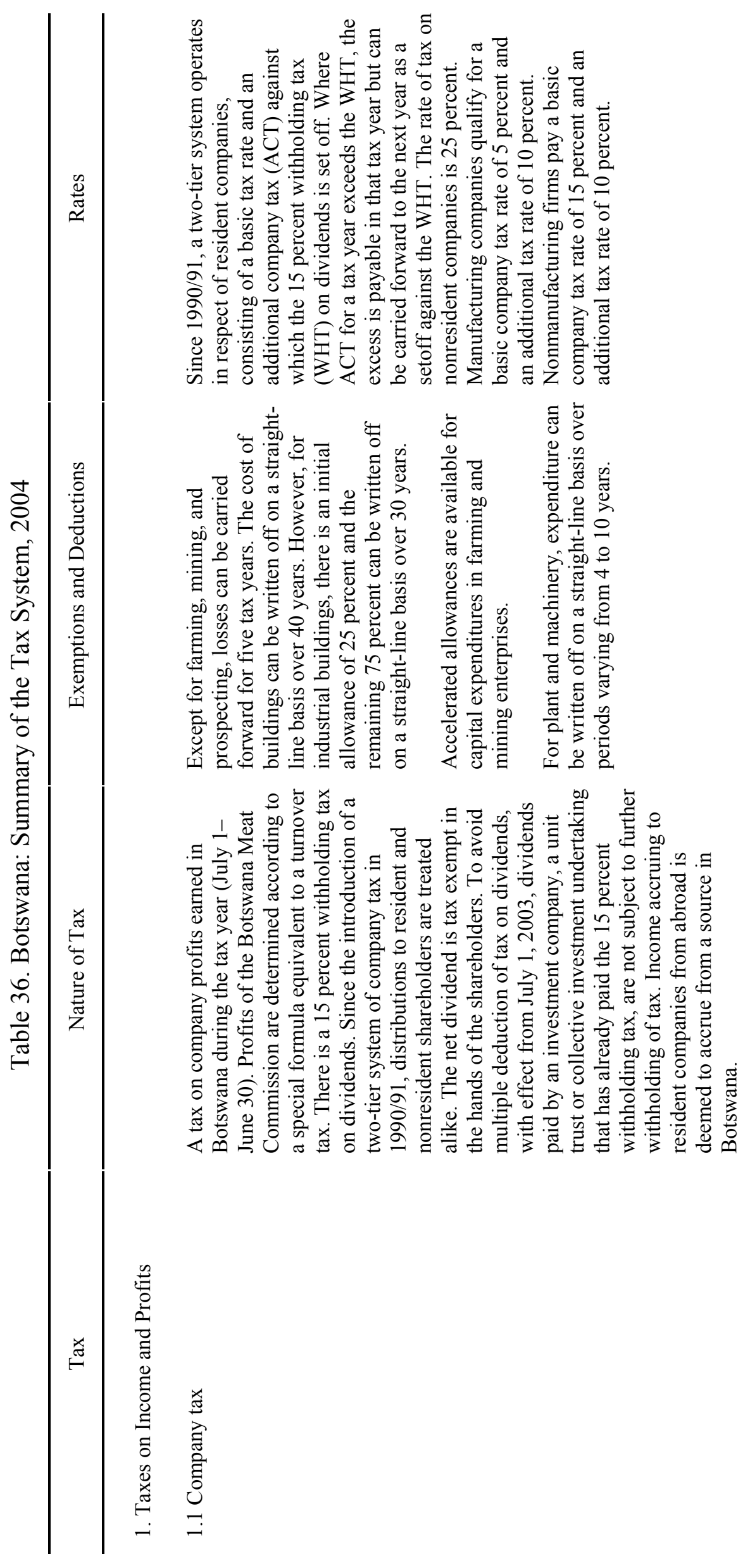




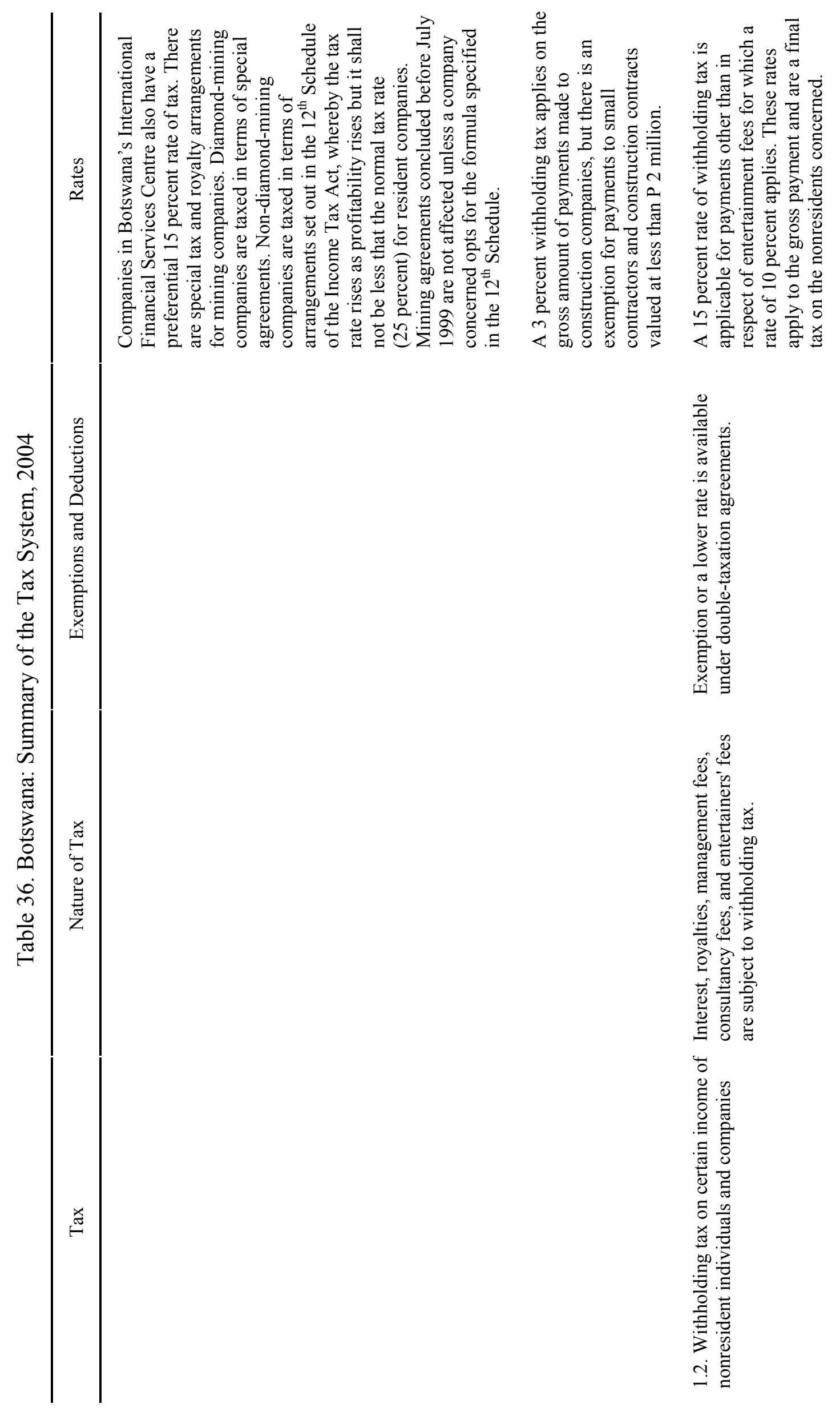




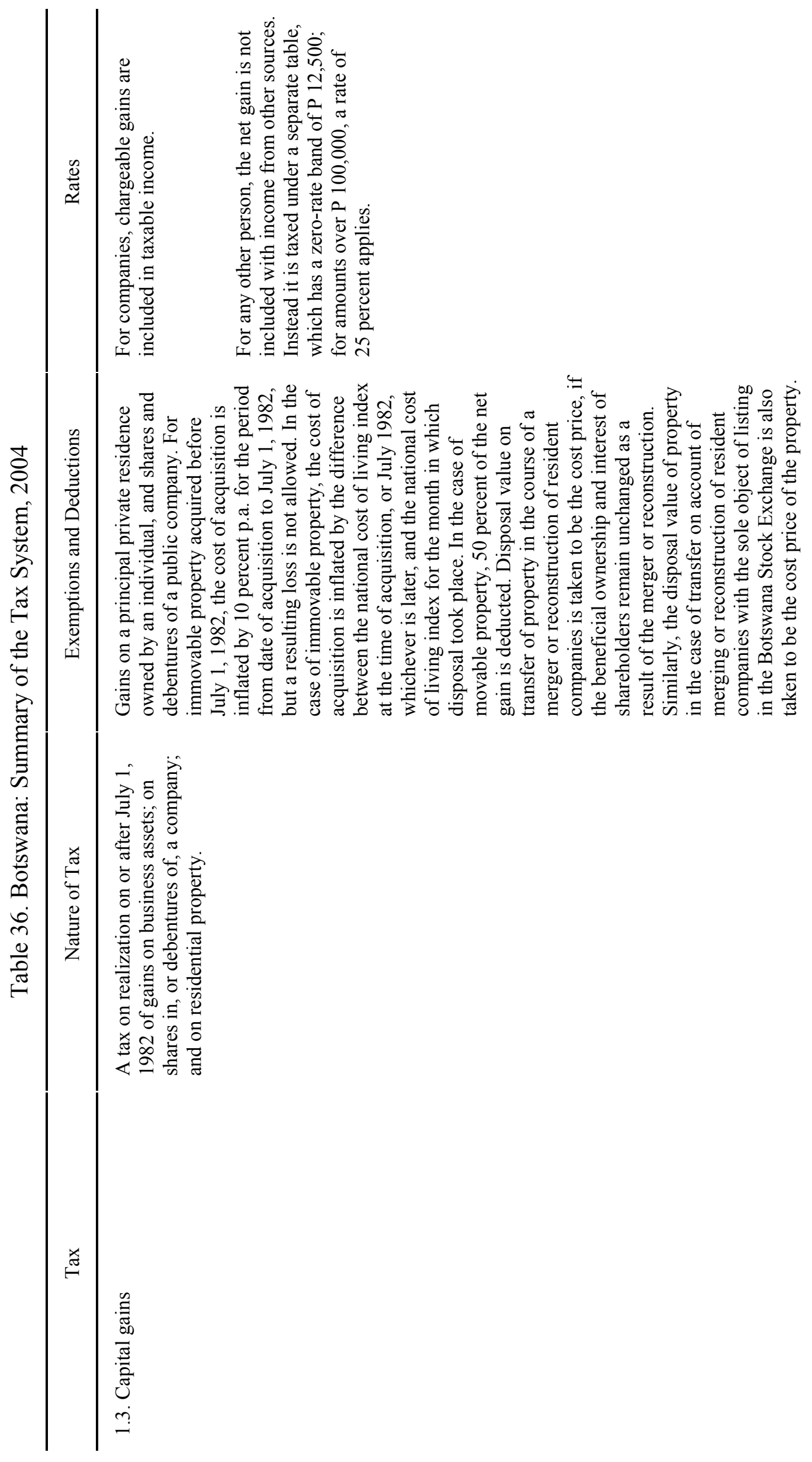




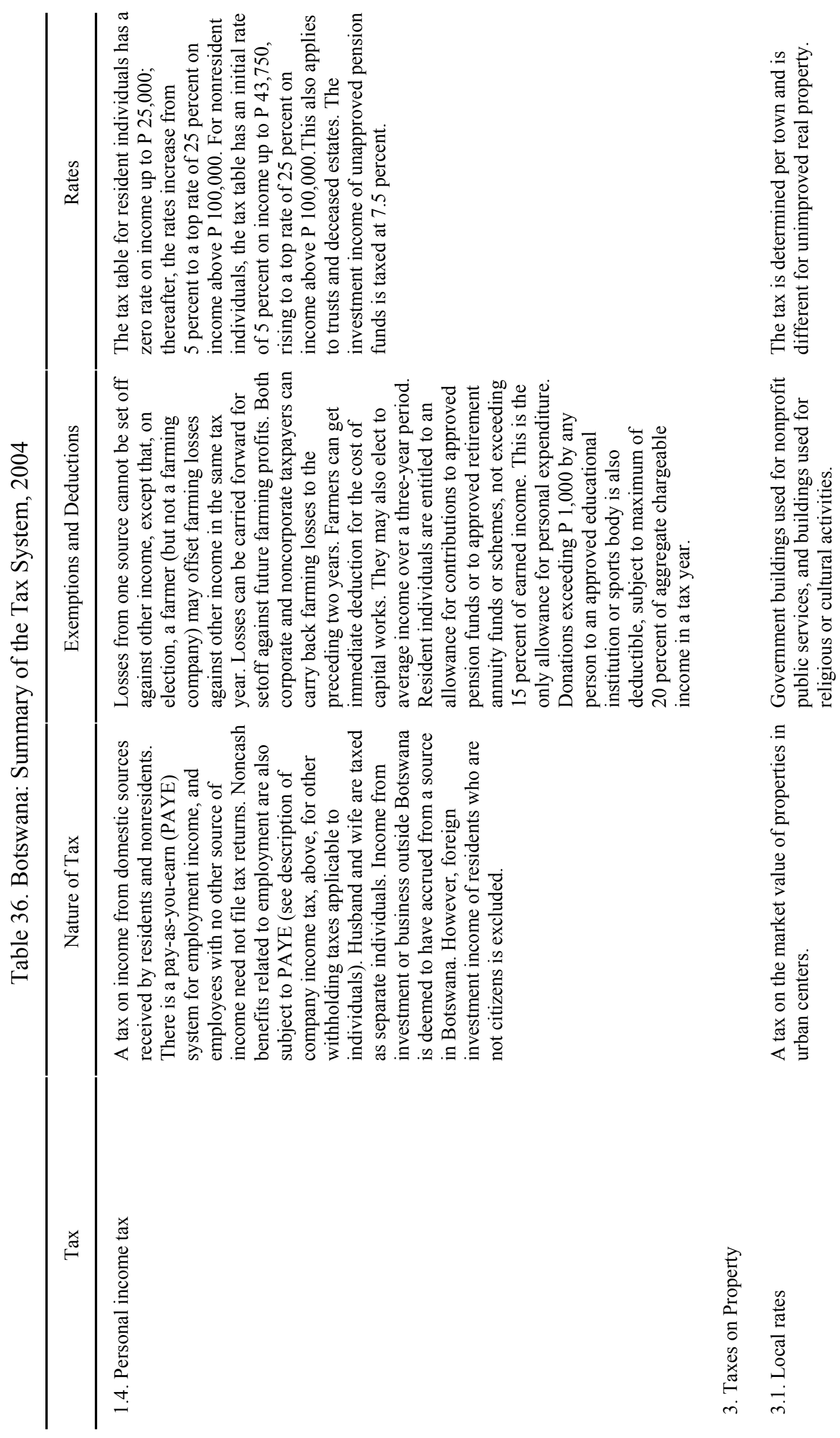




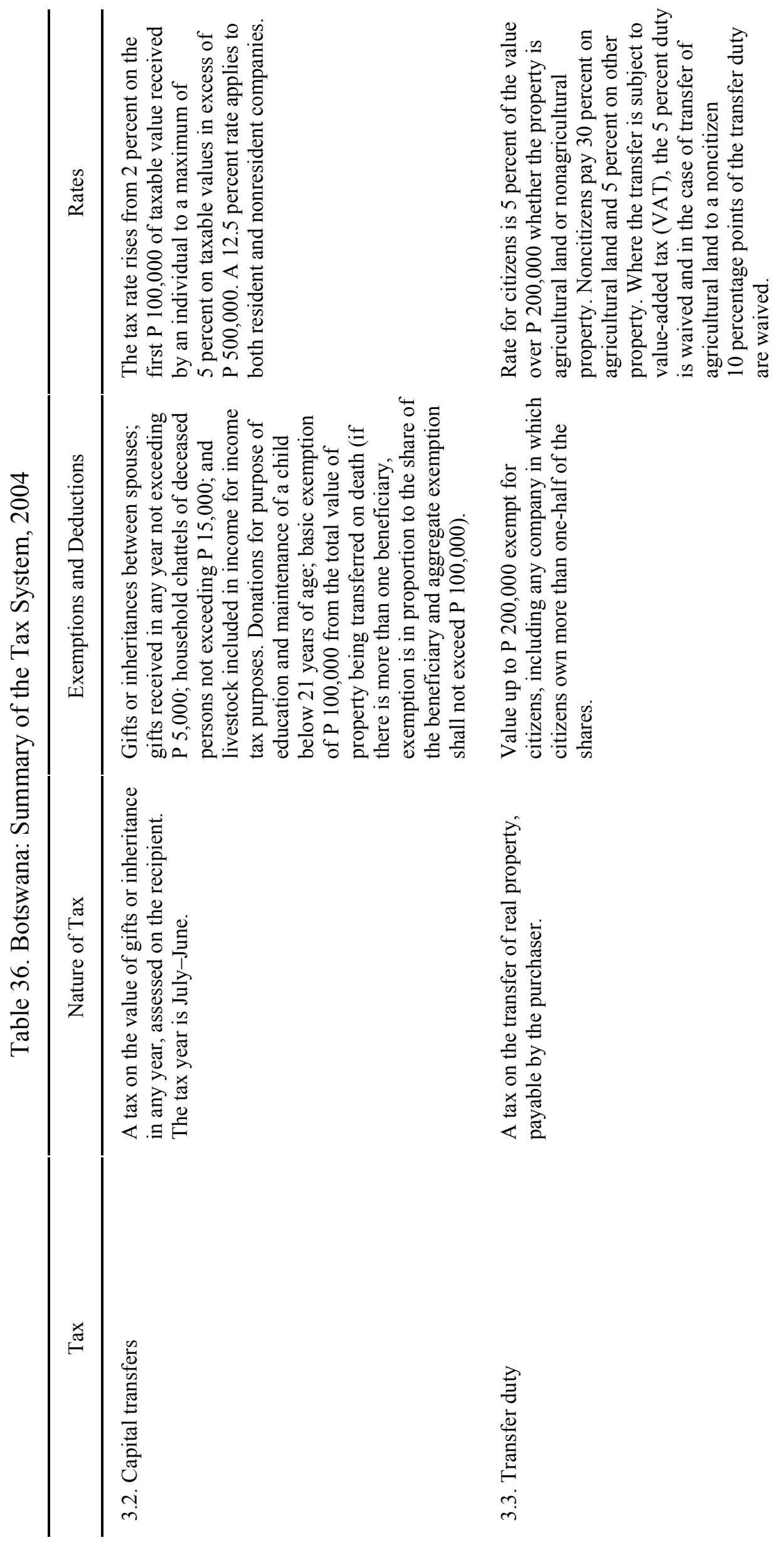




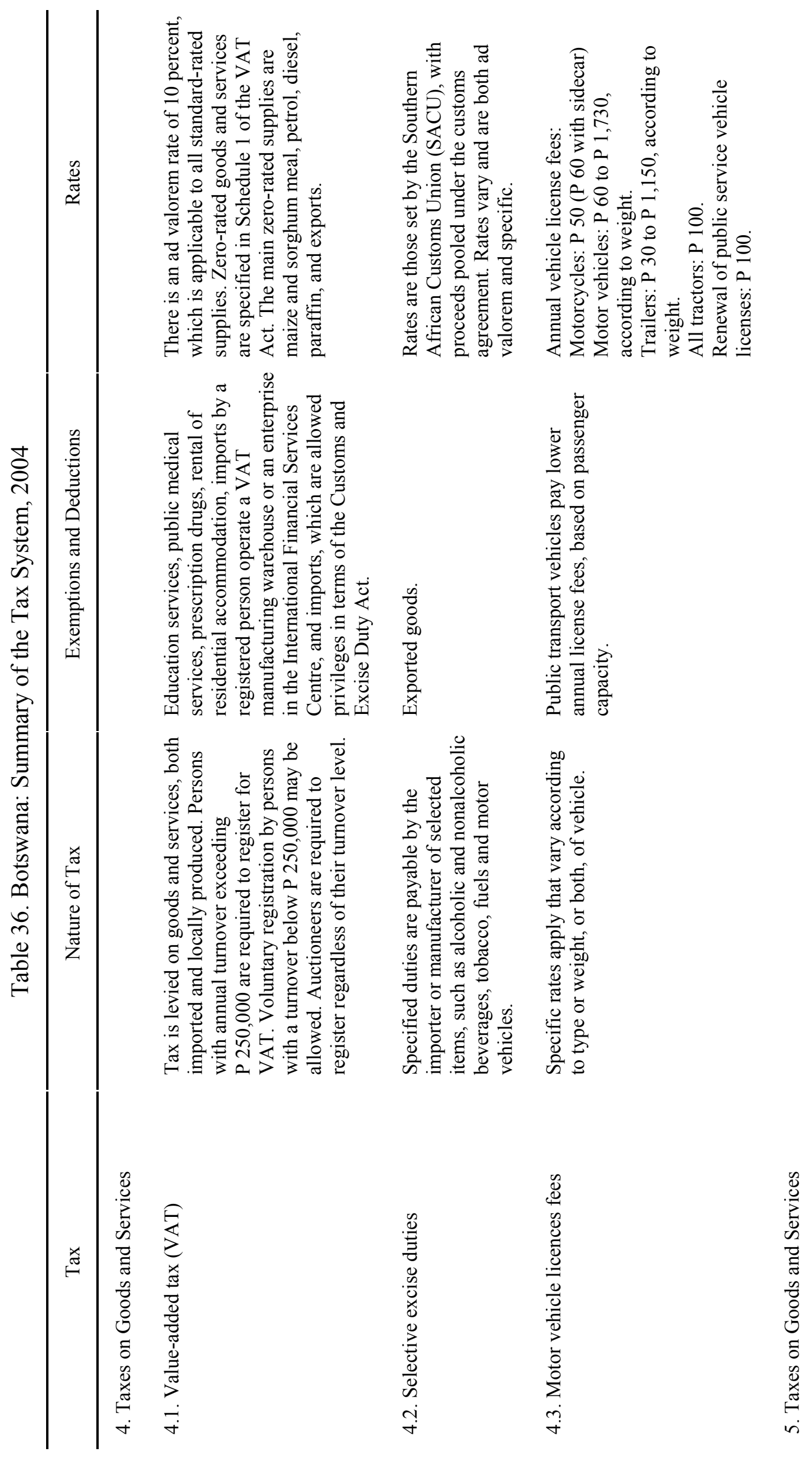




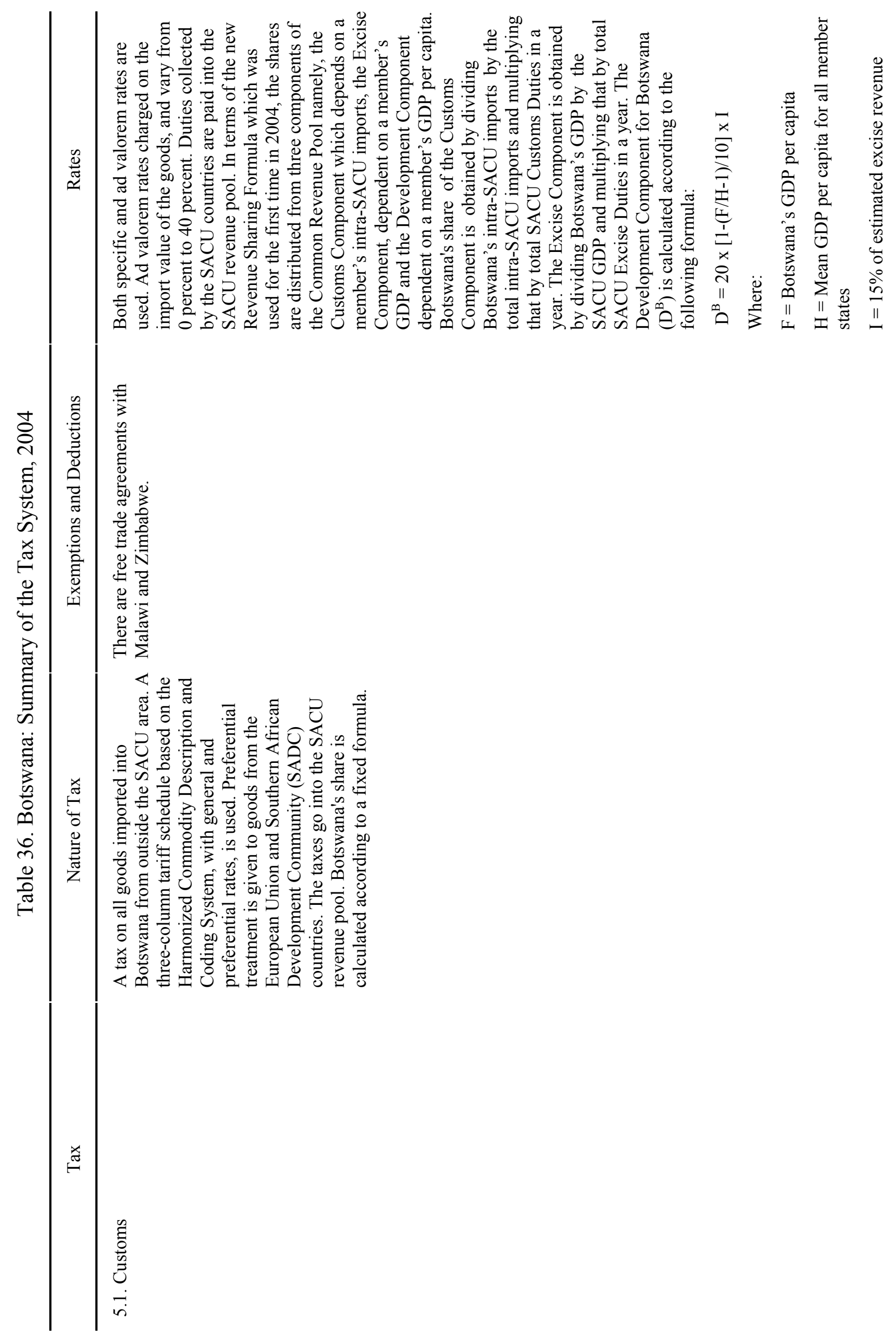


- 46 -

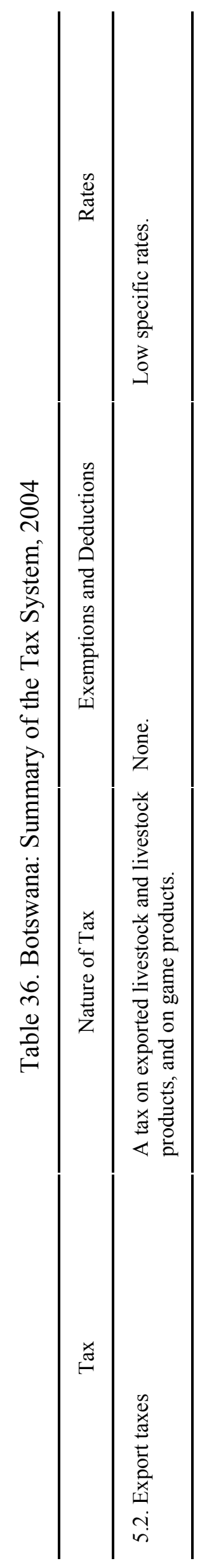

